\title{
第 Springer
}

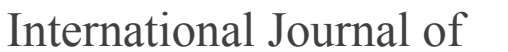

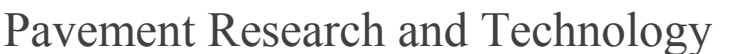

Journal homepage: www.springer.com/42947

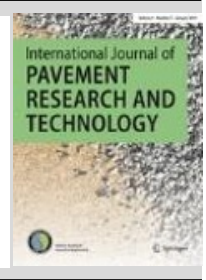

$\square$

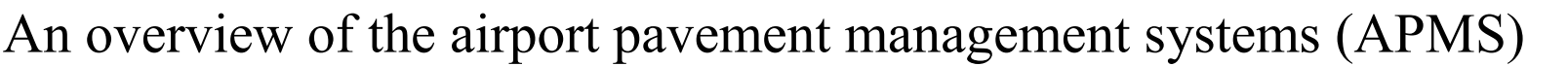

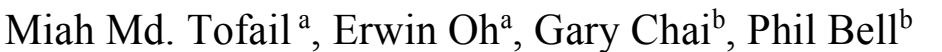

\author{
School of Engineering and Built Environment, Griffith University, Gold Coast, Queensland, Australia \\ Airport Consultancy Group, Gold Coast, Queensland, Australia
}

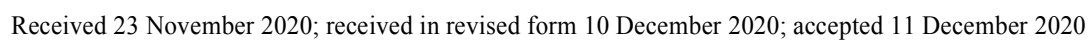

\begin{abstract}

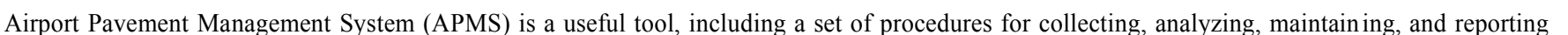

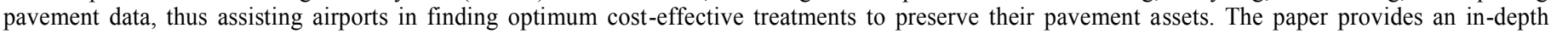

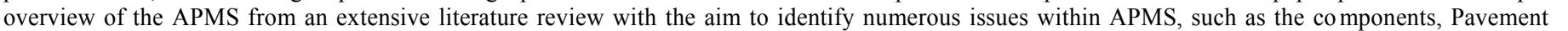

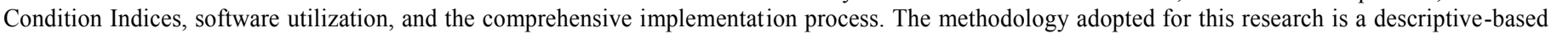

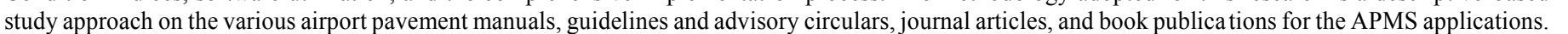

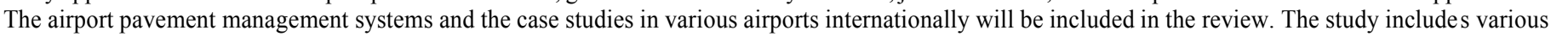

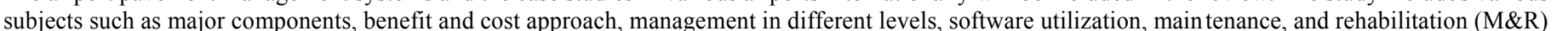

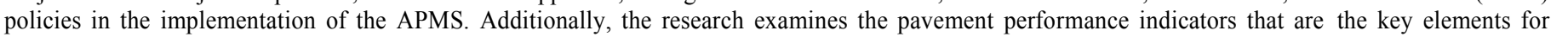

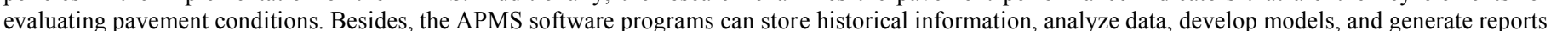

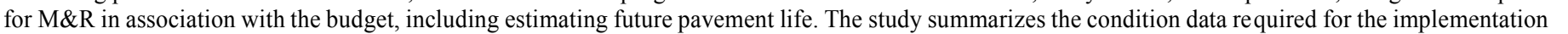

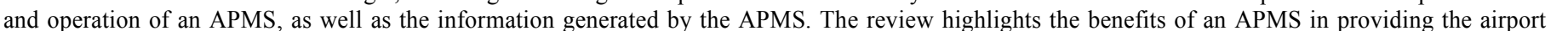

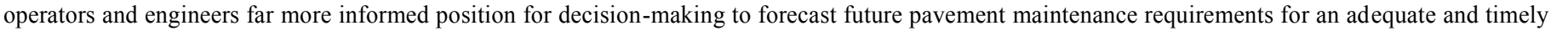
प्राण

$\square$
\end{abstract}

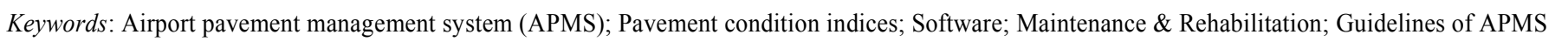
$\square$

\section{Introduction}

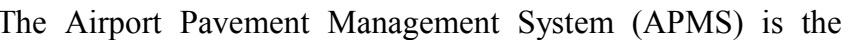

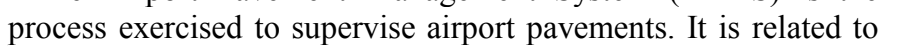

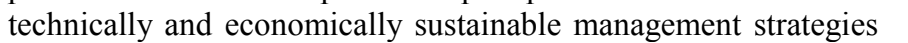
पा

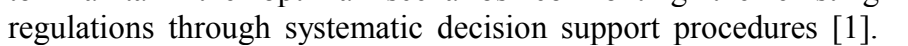

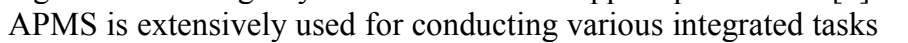

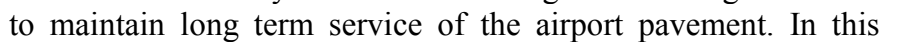

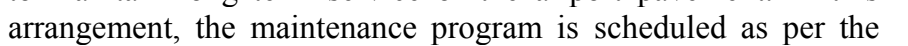

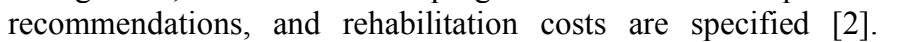

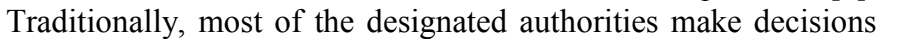

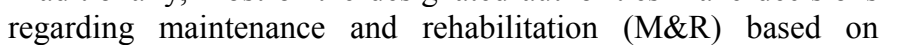

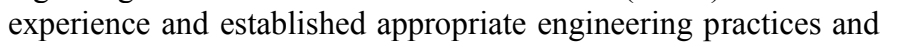

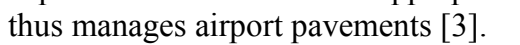

\section{$\square$

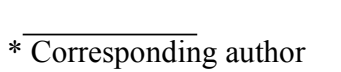

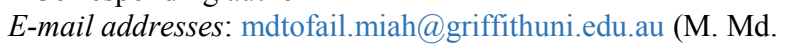

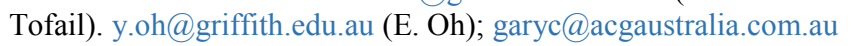

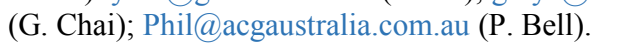

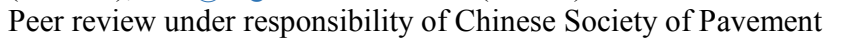

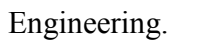

$\square$

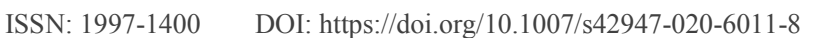

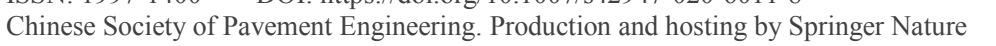

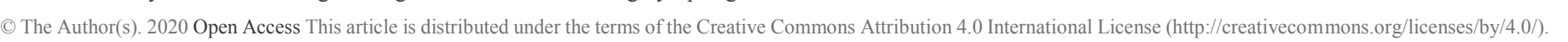

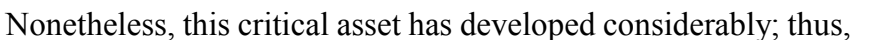

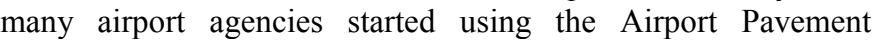

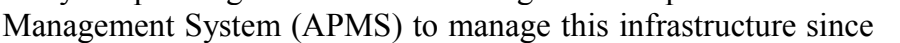

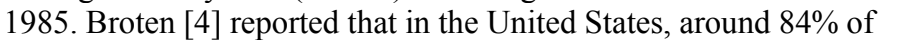

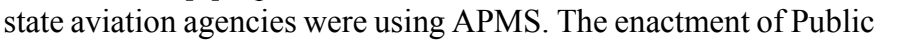

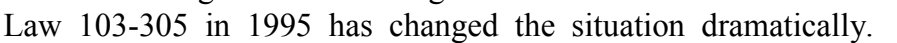

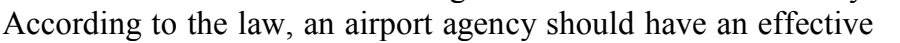

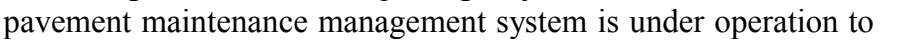

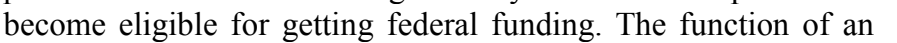

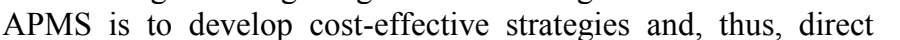

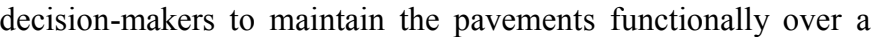

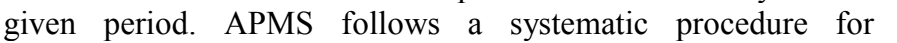

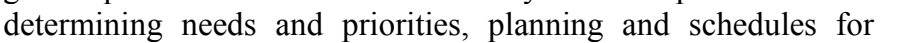

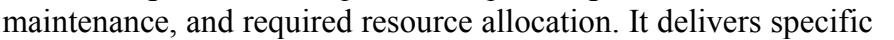

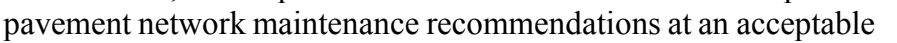

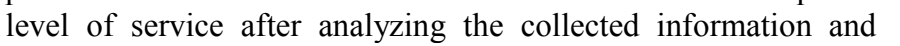

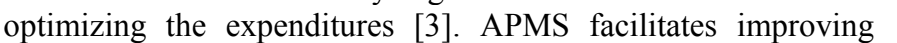

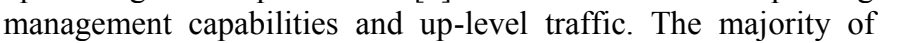

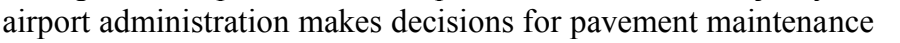

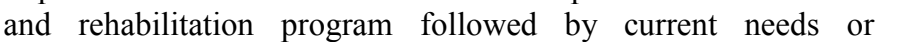

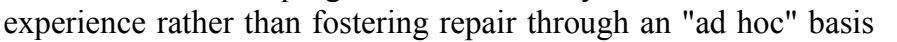
$\mathbb{1 1 0}$ 


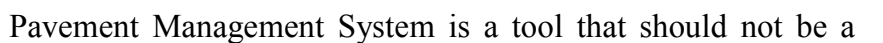

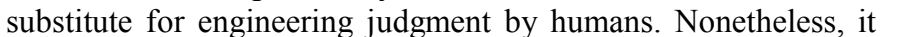

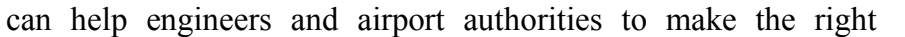

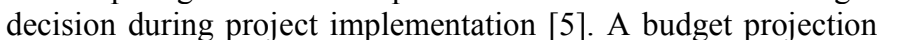

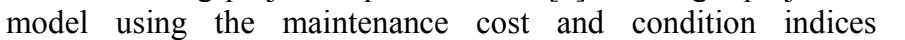

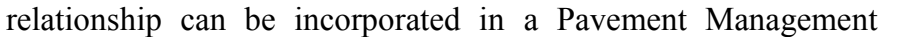

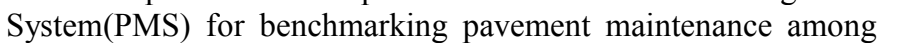

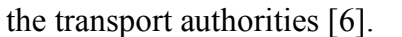

\section{Research objectives}

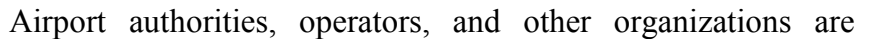

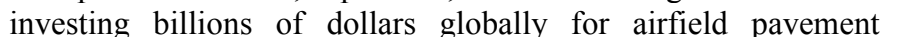

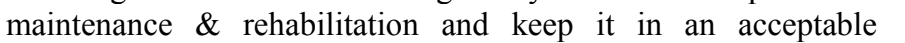

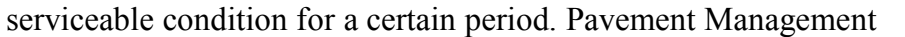

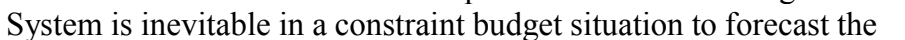

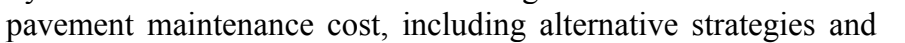

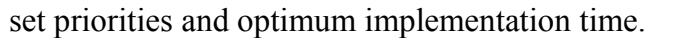

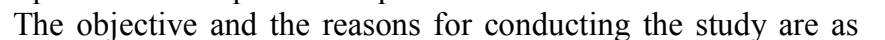

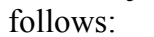

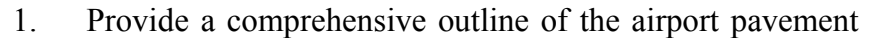

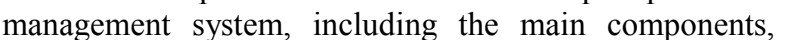

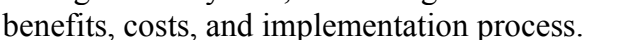

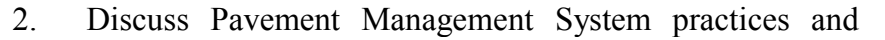

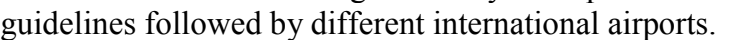

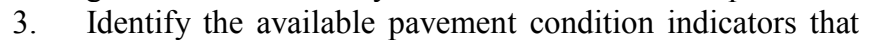

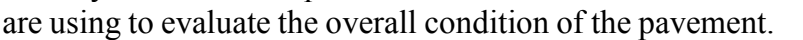

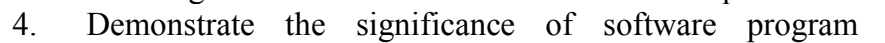

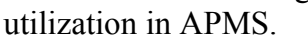

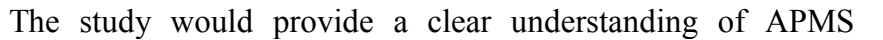

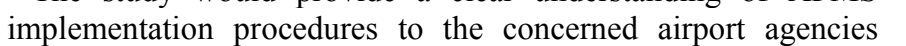

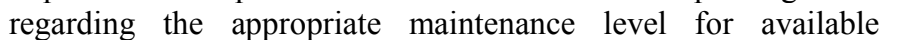
घाण

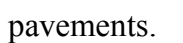

\section{Benefits and costs of airport pavement management}

\subsection{Advantages of APMS}

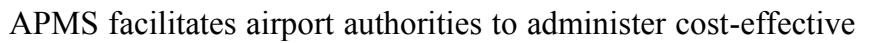

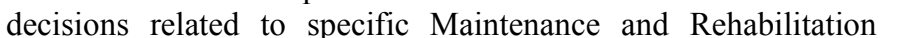

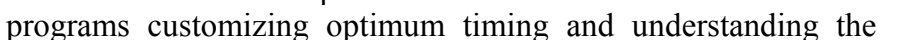
யா

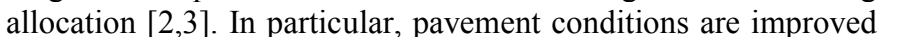

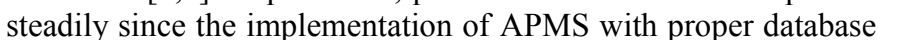

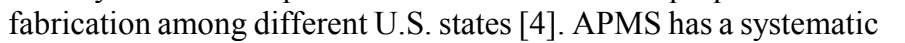

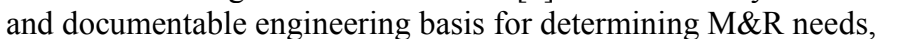

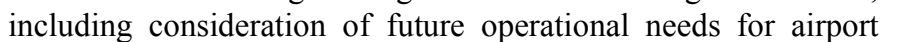

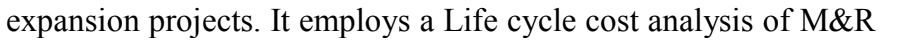

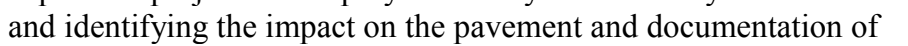

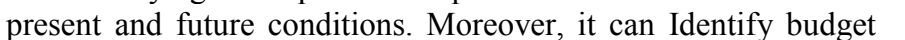

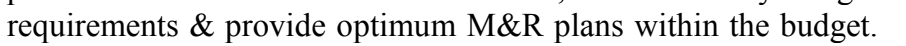

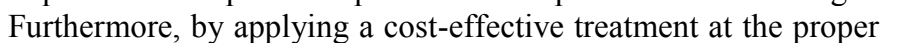

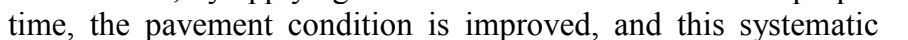

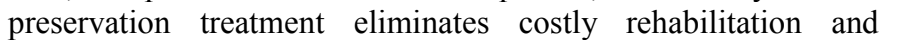

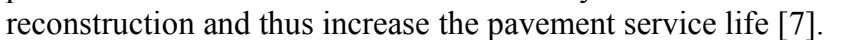

\subsection{Costs involved with APMS}

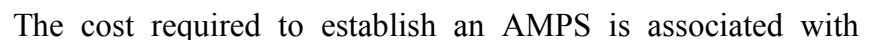

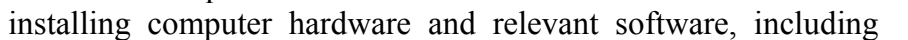

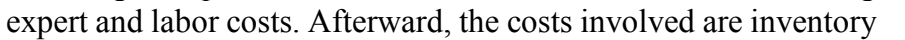

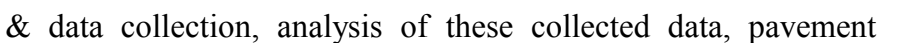

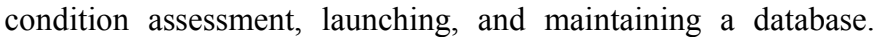

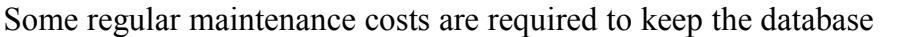

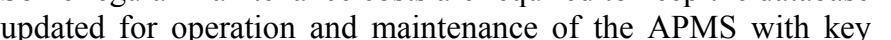

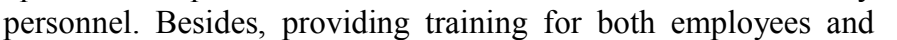
प

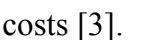

\section{Components in work levels and approaches of APMS}

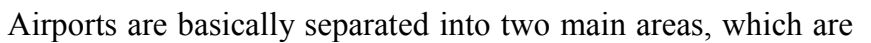

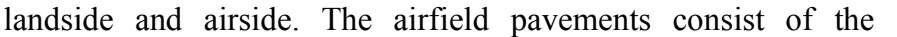

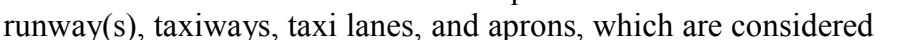

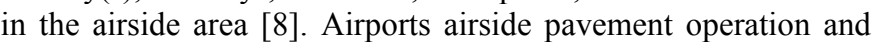

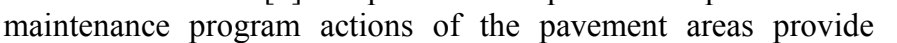

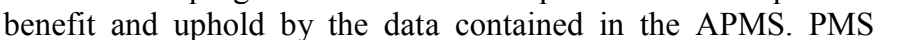

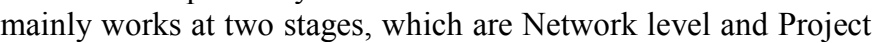

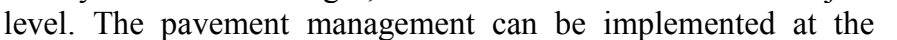

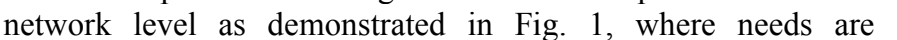

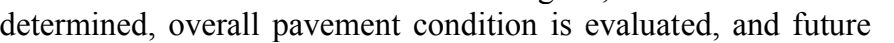

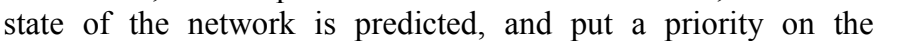

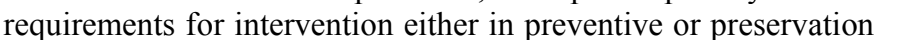

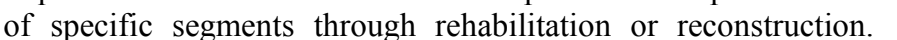

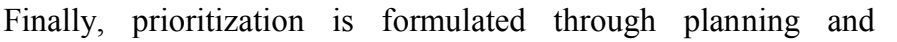

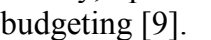

\subsection{Airport pavement management in network and project level}

\subsubsection{Network level management}

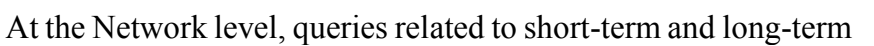

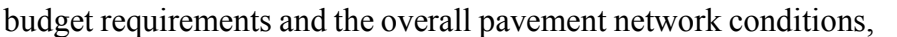

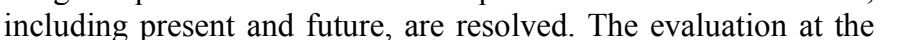

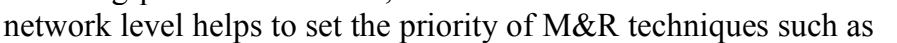

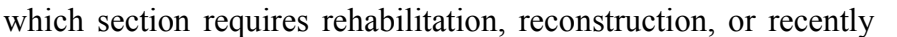

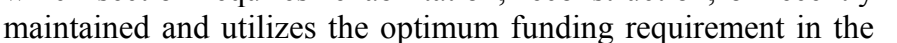

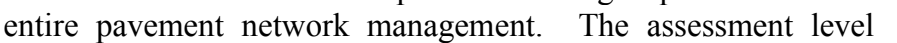

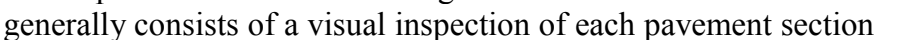

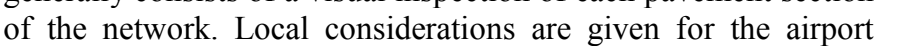

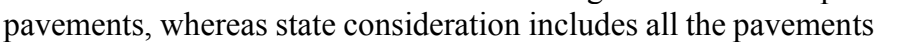

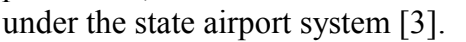

\subsubsection{Project level management}

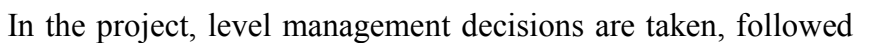

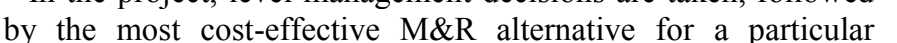

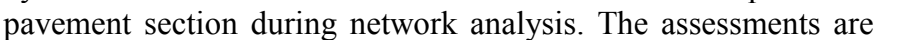

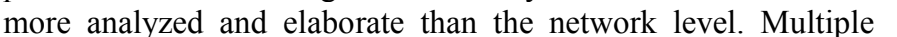

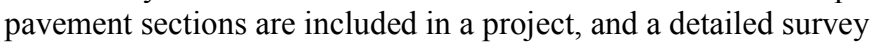
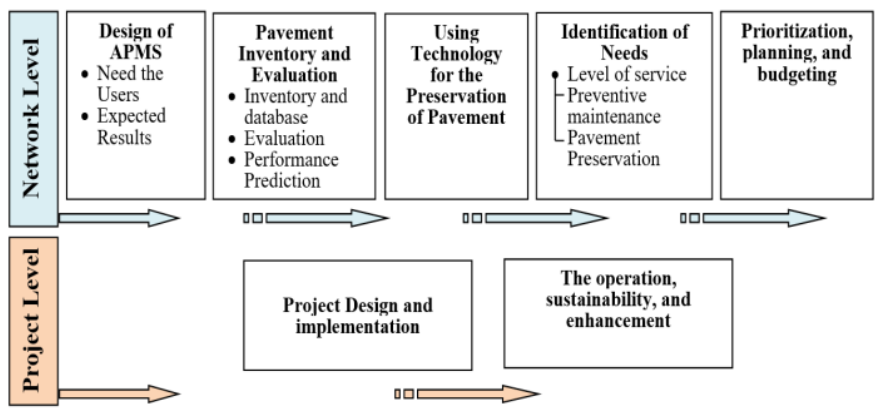

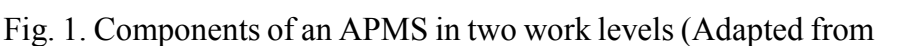
पा1⿴囗十ा 


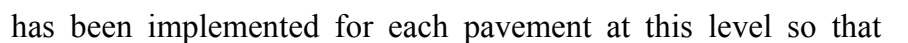

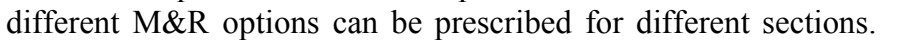

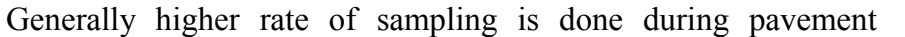

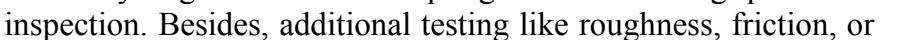

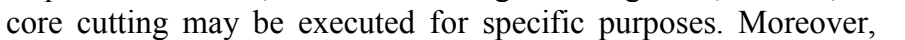

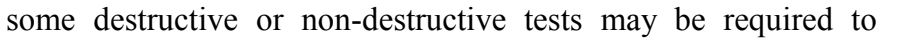

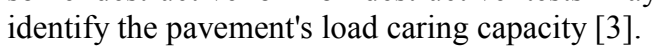

\subsection{Other management levels}

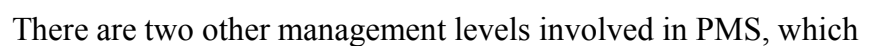

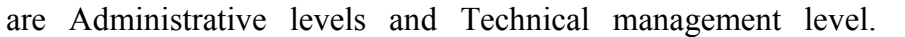

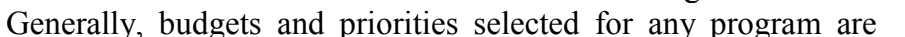

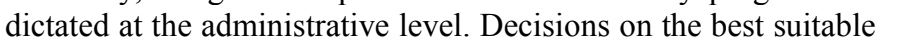

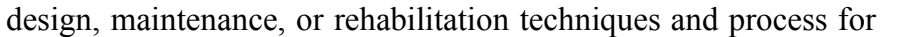

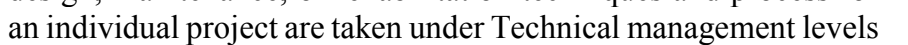
पाणा।|णा

\subsection{Approaches to airport pavement maintenance and rehabilitation in the traditional system:}

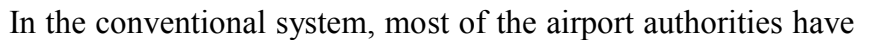

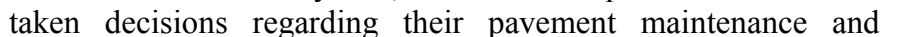

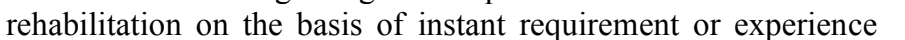

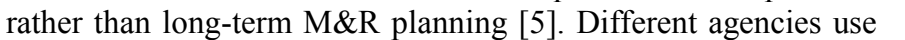

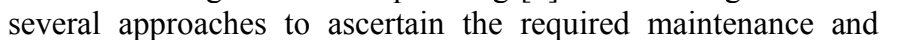

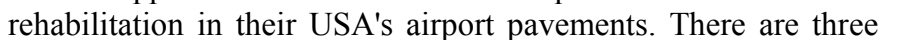

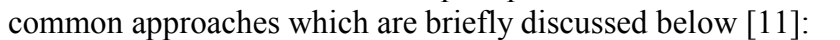

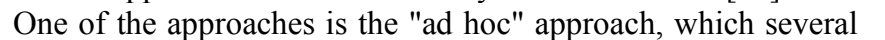
agencies frequently take. The agency's workforce implements

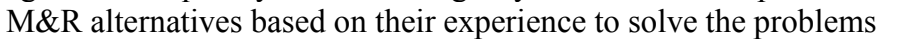

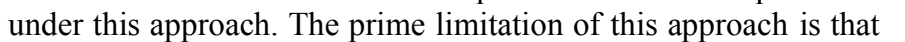
पा

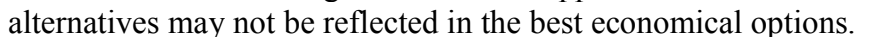

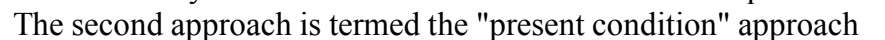

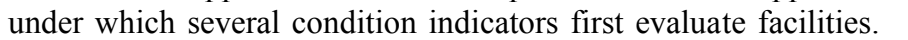

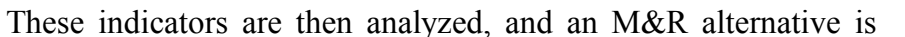

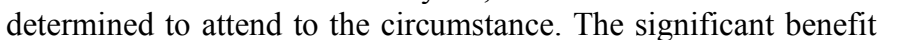

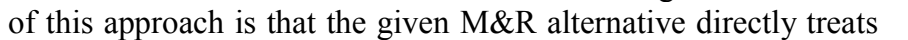

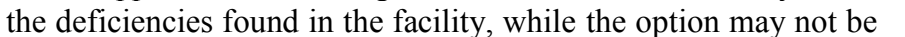

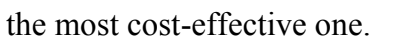

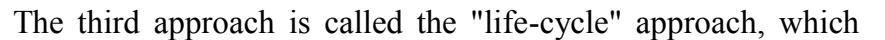

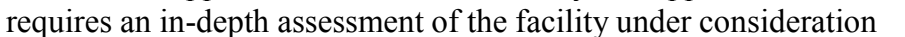

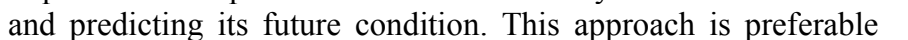

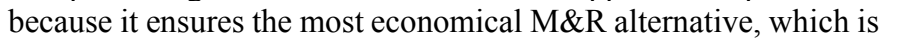

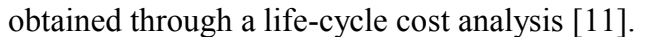

\section{Airport pavement management system implementations}

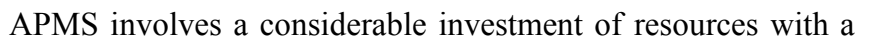

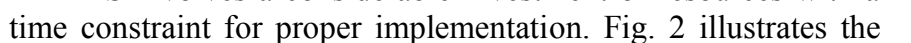

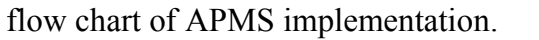

\subsection{Potential users and their requirement}

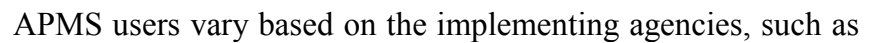

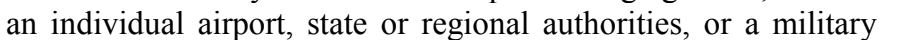

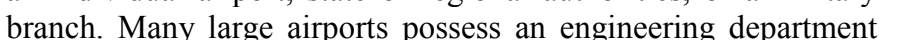

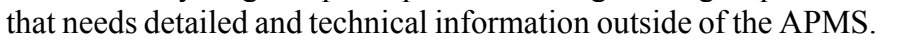

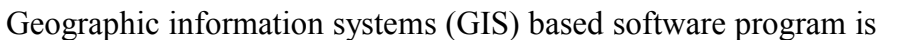

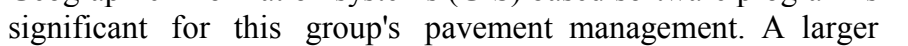

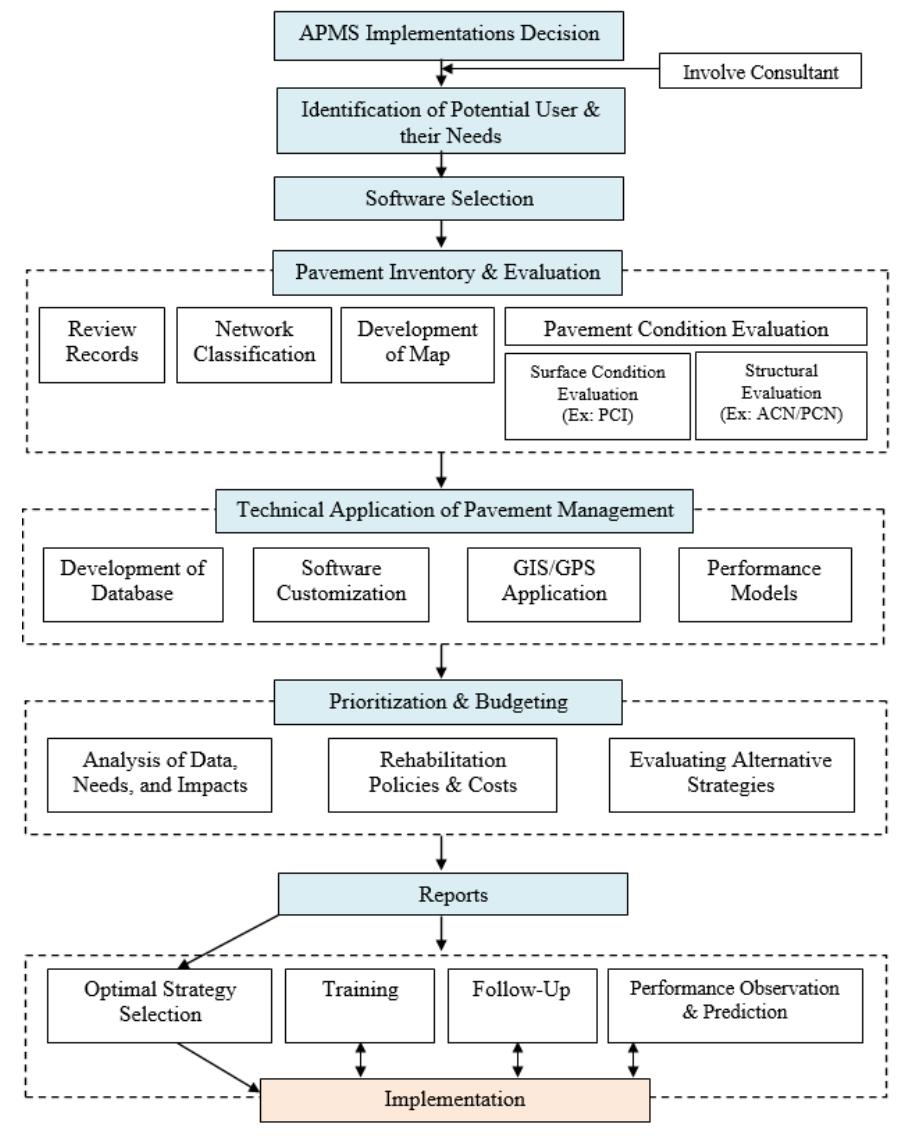

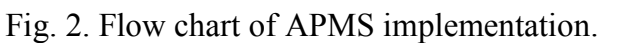

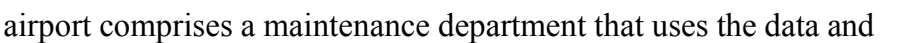
एणी

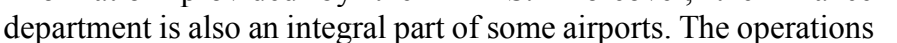

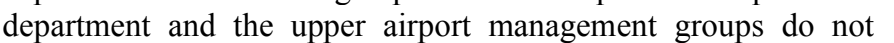

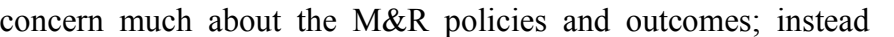

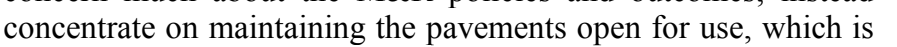

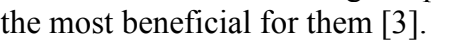

\subsection{Software selection}

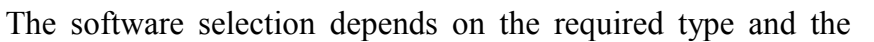

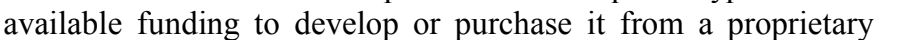

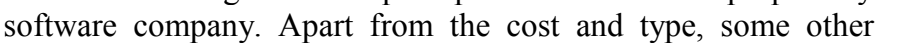

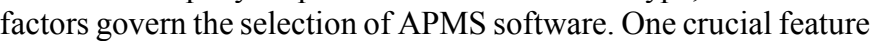

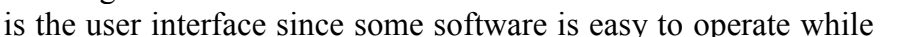

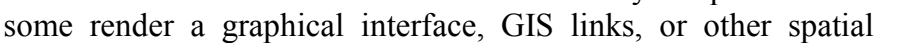

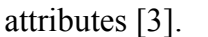

\subsection{Pavement inventory and evaluation}

\subsubsection{Review of the records}

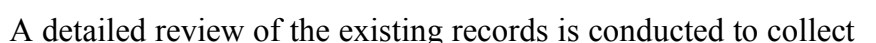

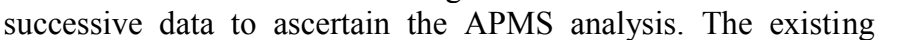

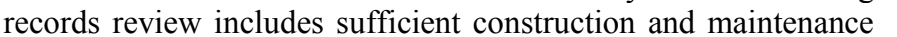

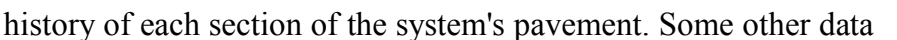

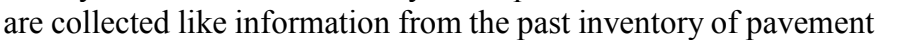

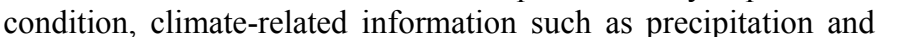

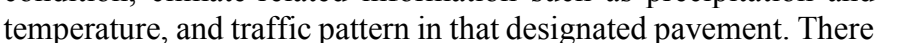

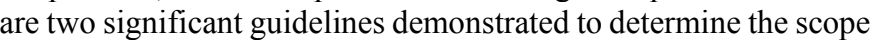




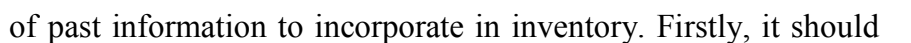

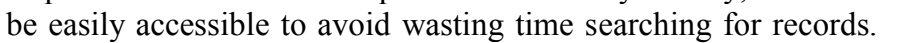

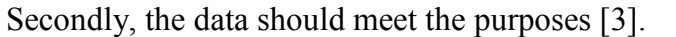

\subsubsection{Network classification}

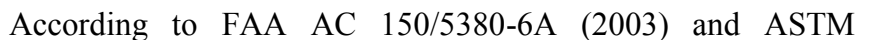

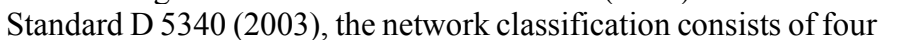

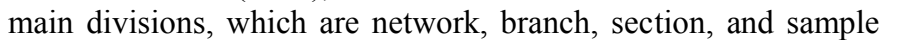

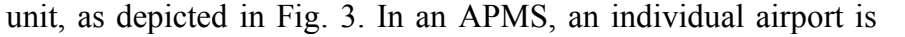

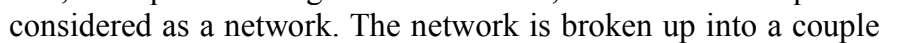

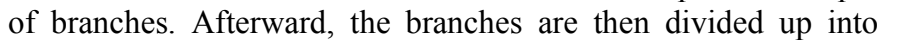

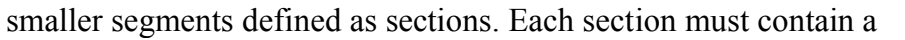

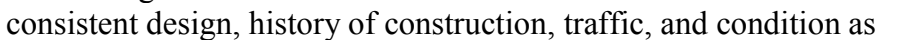

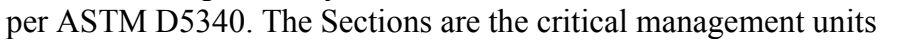

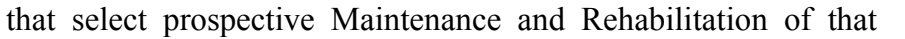

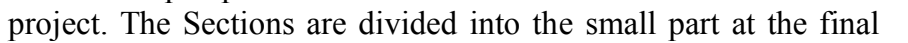

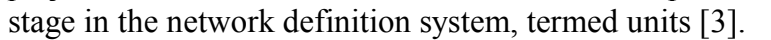

\subsubsection{Development of map}

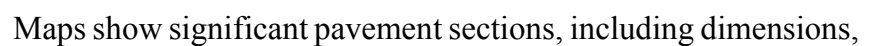

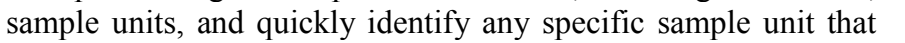

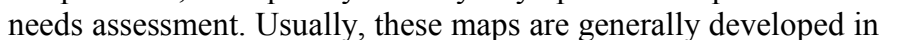

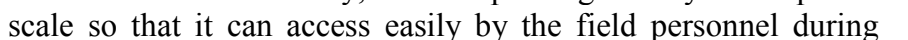

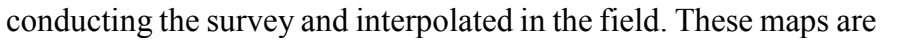

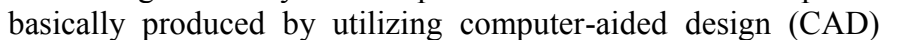

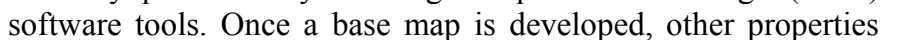

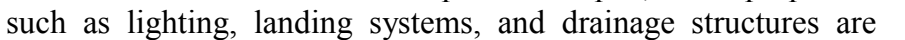

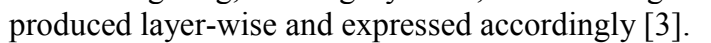

\subsubsection{Evaluation of pavement}

\section{Surface condition evaluation}

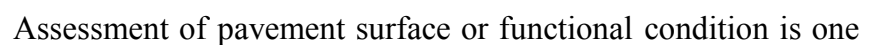

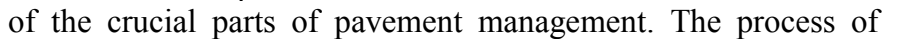

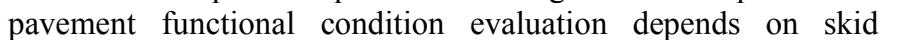

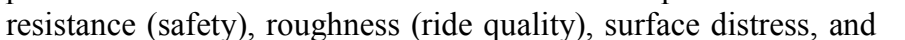

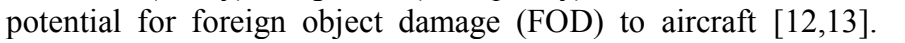

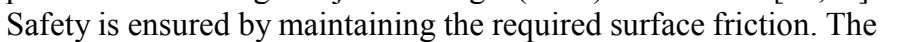
evaluation of a pavement's surface condition allows deciding

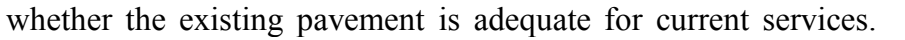

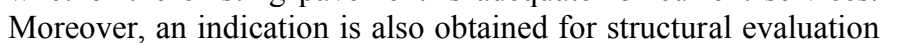
एம

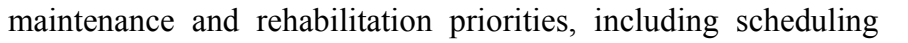

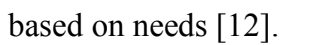

\section{Pavement condition indices}

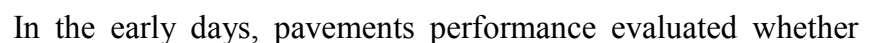
एம

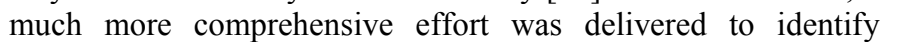

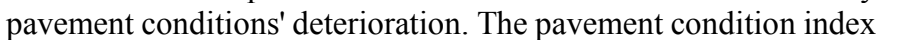

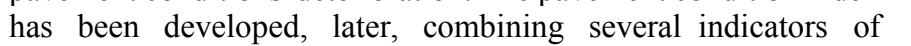

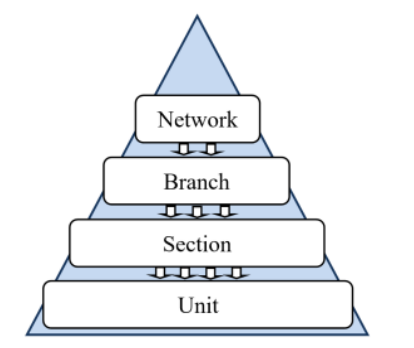

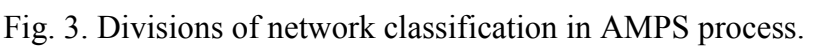

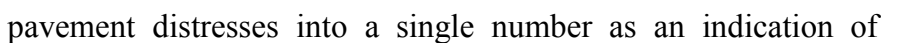

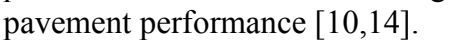

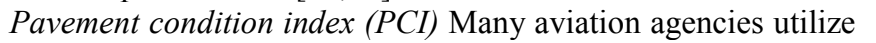

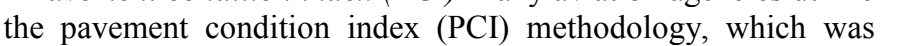

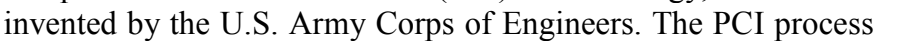

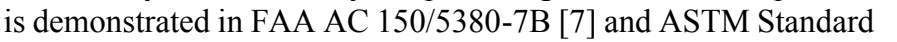

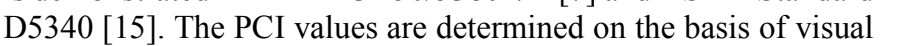

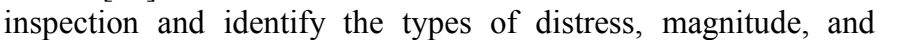

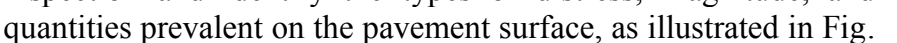

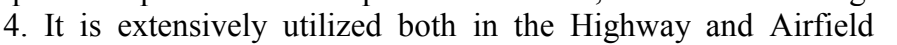

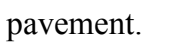

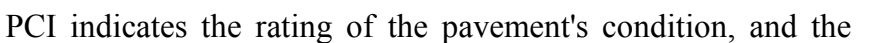

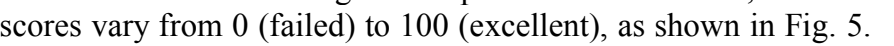

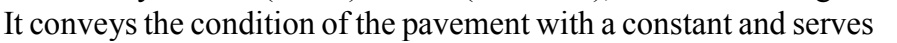

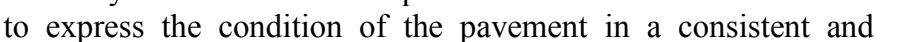

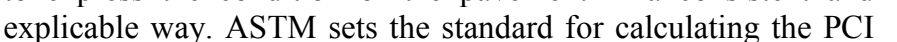

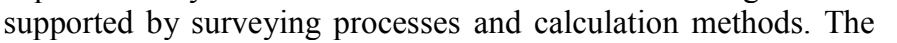

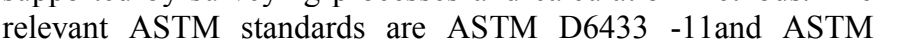

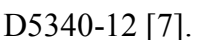

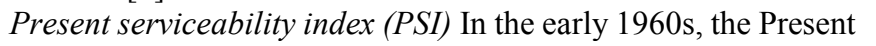

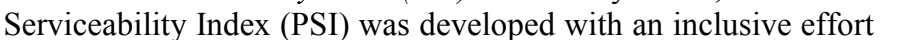

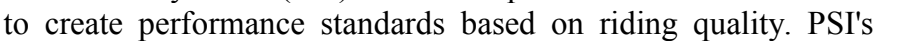

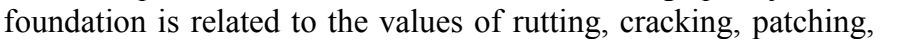

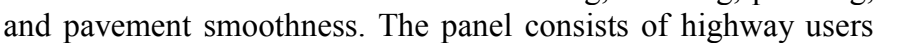

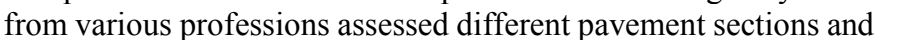

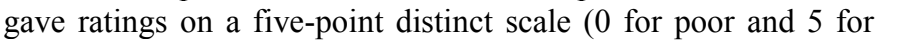

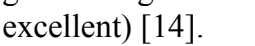

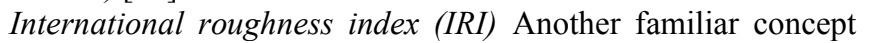

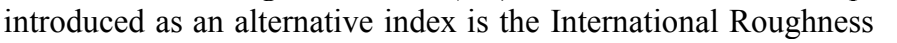

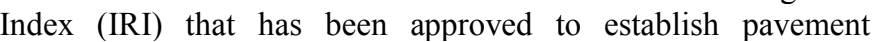

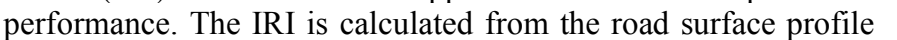

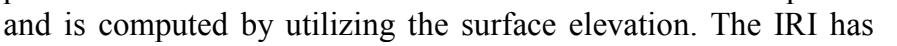

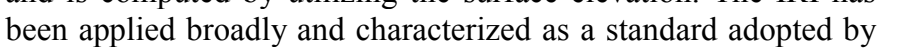

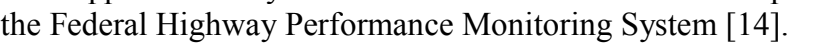

\section{Structural ability evaluation}

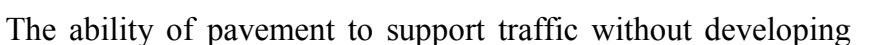

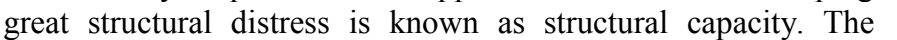

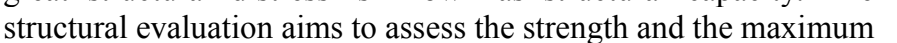

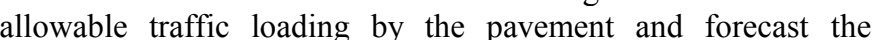

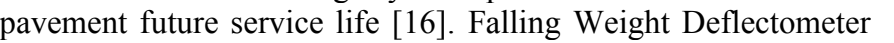

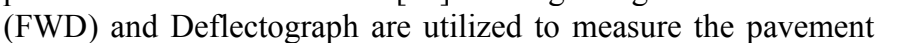

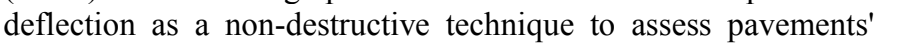

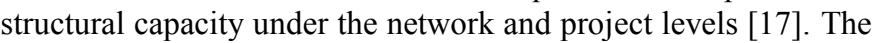

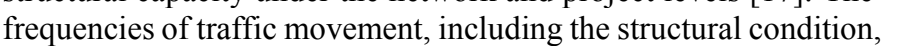

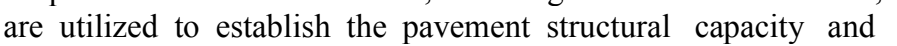

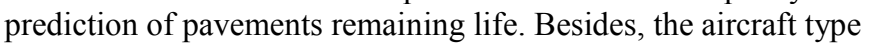

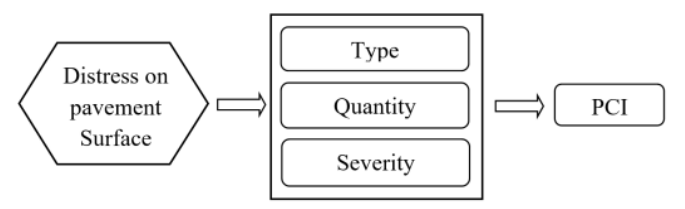

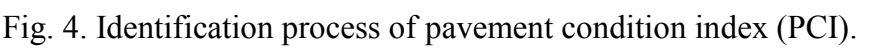

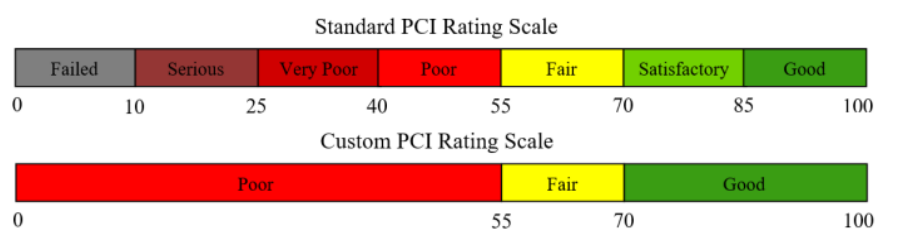

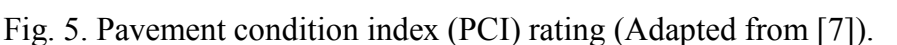




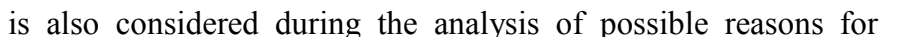

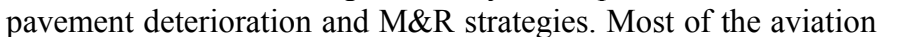

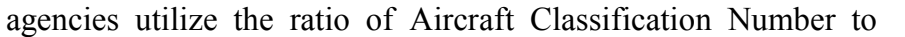

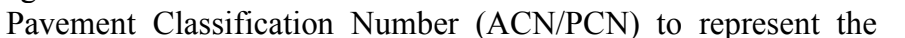

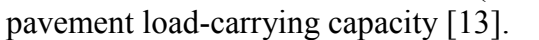

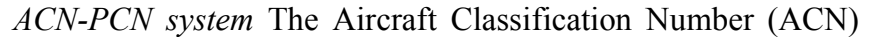

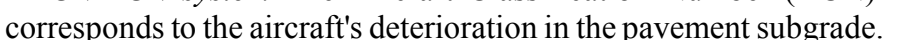

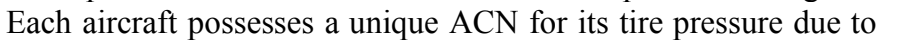

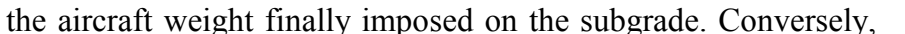

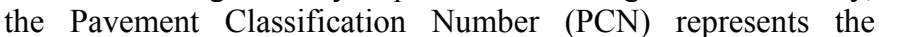

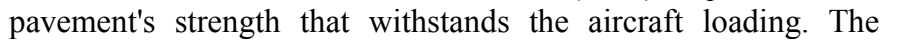

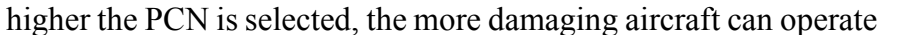

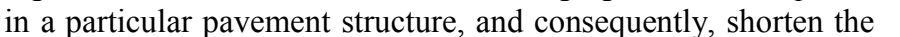

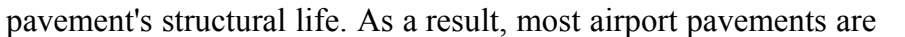

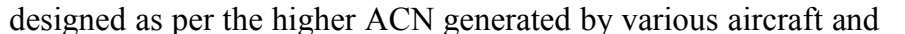

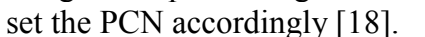

\section{Functional requirements}

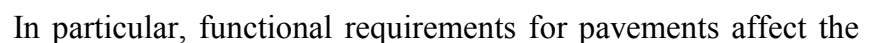

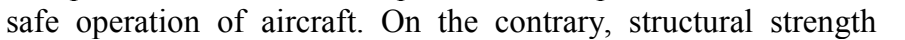

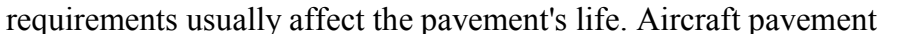

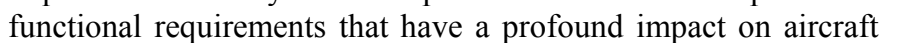

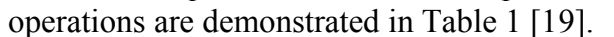

\section{Frequency of inspection and thresholds:}

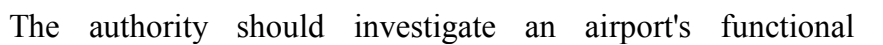

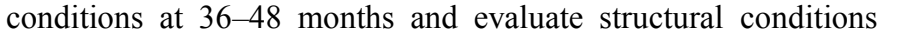

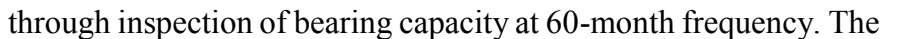

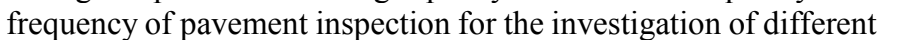
पा

पालाणा

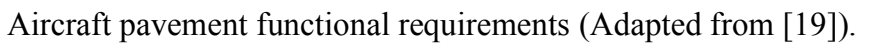

\begin{tabular}{|c|c|c|c|c|}
\hline \multirow[t]{2}{*}{ 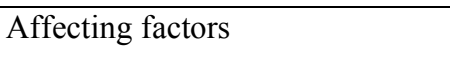 } & \multicolumn{4}{|c|}{ 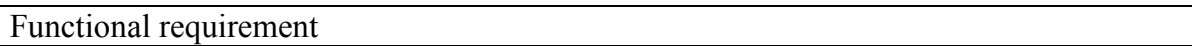 } \\
\hline & 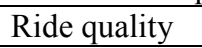 & 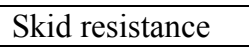 & 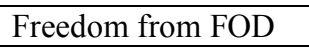 & 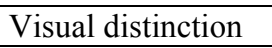 \\
\hline 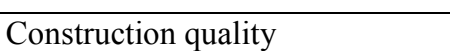 & $\square \square \square$ & $\square$ & $\square$ & $\square$ \\
\hline 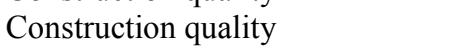 & $\square$ & $\square$ & $\square \square \mathbb{Q}$ & $\square$ \\
\hline 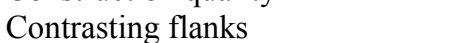 & $\square$ & $\square$ & $\square$ & $\square \square \mathbb{Q}$ \\
\hline 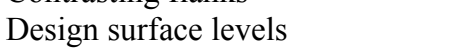 & $\square \square \square$ & $\square$ & $\square$ & $\square$ \\
\hline 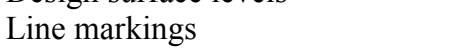 & $\square$ & $\square$ & $\square$ & $\square \square \square$ \\
\hline 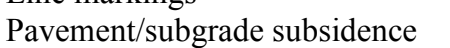 & $\square \square \square$ & $\square$ & $\square$ & $\square$ \\
\hline 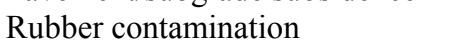 & $\square$ & $\square \square \mathbb{Q}$ & $\square$ & $\square$ \\
\hline 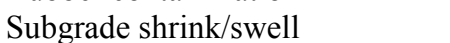 & $\square \square \mathbb{Q}$ & $\square$ & $\square$ & $\square$ \\
\hline 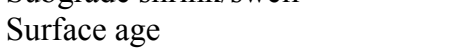 & $\square$ & $\square \square \square$ & $\square$ & $\square$ \\
\hline 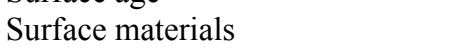 & $\square$ & $\square \square \square$ & $\square$ & $\square$ \\
\hline 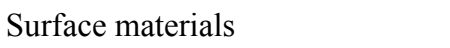 & $\square$ & $\square$ & $\square \square \square$ & $\square$ \\
\hline 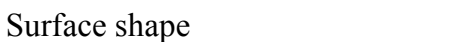 & $\square$ & $\square \square \square$ & $\square$ & $\square$ \\
\hline 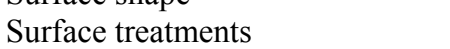 & $\square$ & $\square \square \square$ & $\square$ & $\square$ \\
\hline 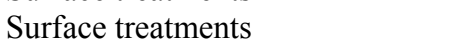 & $\square$ & $\square$ & $\square \square \square$ & $\square$ \\
\hline 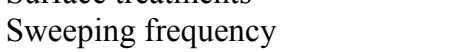 & $\square$ & $\square$ & $\square \square \mathbb{Q}$ & $\square$ \\
\hline 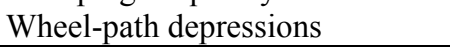 & $\square \square \square$ & $\square$ & $\square$ & $\square$ \\
\hline
\end{tabular}

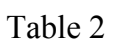

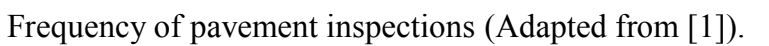

\begin{tabular}{|c|c|c|c|}
\hline \multirow[t]{2}{*}{ एण1⿴囗十 } & \multicolumn{2}{|c|}{ 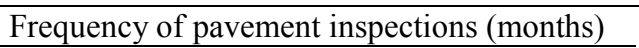 } & \multirow[t]{2}{*}{ 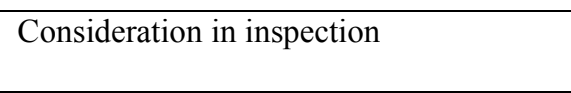 } \\
\hline & 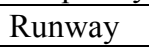 & 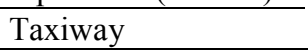 & \\
\hline$\square \square \square$ & $\square \square$ & $\square \square$ & 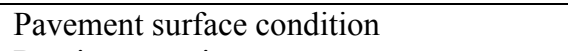 \\
\hline$\square \square \square \square$ & $\square \square$ & $\square \square$ & 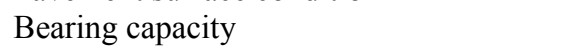 \\
\hline प⿴囗十丁 & $\square$ & $\square \square$ & 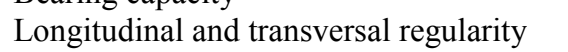 \\
\hline$\square \square \square \square$ & $\square \square$ & $\square \square$ & 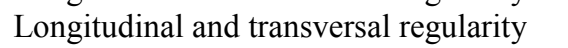 \\
\hline$\square \square \square \square$ & $\square \square$ & 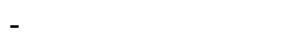 & 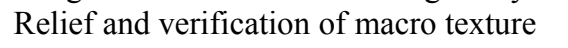 \\
\hline$\square \square \square \square$ & $\square \square$ & $\square \square$ & 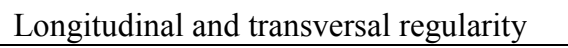 \\
\hline
\end{tabular}

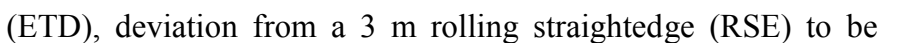

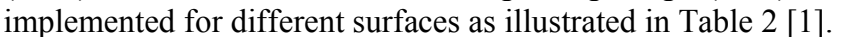

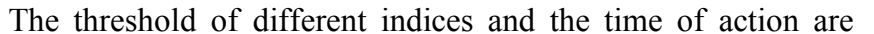

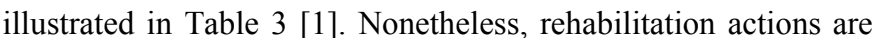

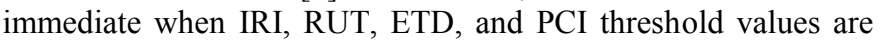

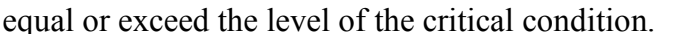

\subsection{Technological application of pavement maintenance \& management}

\subsubsection{Development of database}

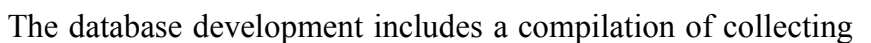

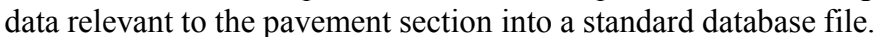

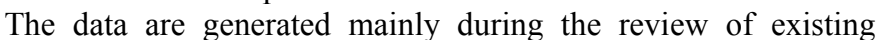

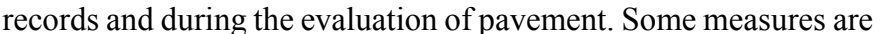

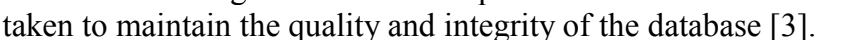

\subsubsection{Customization of software}

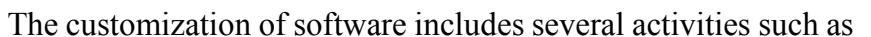

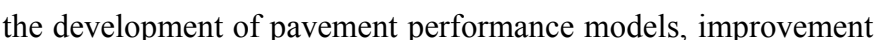

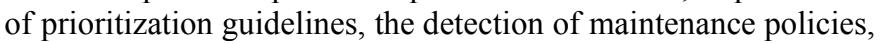

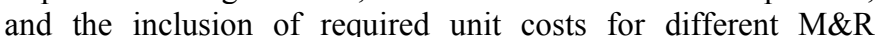

\subsubsection{Using instruments in pavements}

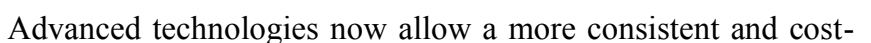

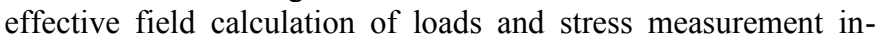

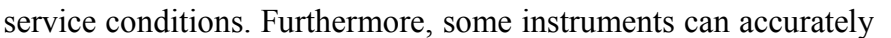

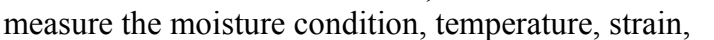

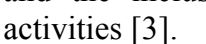




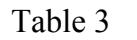

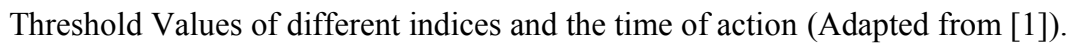

\begin{tabular}{|c|c|c|c|c|c|c|c|}
\hline \multirow{3}{*}{ 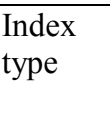 } & \multirow{3}{*}{ 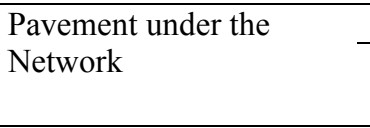 } & \multicolumn{6}{|c|}{ 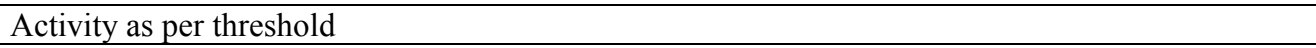 } \\
\hline & & \multirow[t]{2}{*}{ 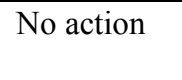 } & \multirow{2}{*}{ 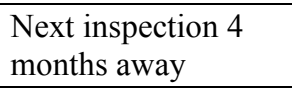 } & \multirow{2}{*}{ 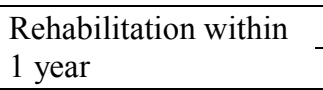 } & \multicolumn{3}{|c|}{ 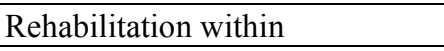 } \\
\hline & & & & & 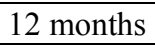 & 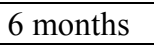 & 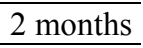 \\
\hline \multirow[t]{2}{*}{ 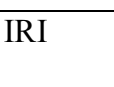 } & 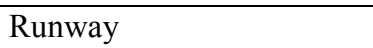 & 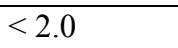 & $2.0 \leq \mathrm{IRI}<2.5 \square$ & $2.5 \leq \mathrm{IRI}<3.0 \square$ & $\square$ & 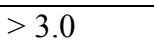 & $\bar{\square}$ \\
\hline & 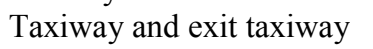 & 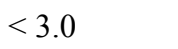 & $3.0 \leq \mathrm{IRI}<3.4 \square$ & $3.4 \leq \mathrm{IRI}<3.8 \square$ & $\square$ & पाणाए & $\square$ \\
\hline$\square \square \square \square$ & 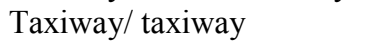 & पणाणा & $12.5 \leq \mathrm{RUT}<25.0 \square$ & $\square$ & 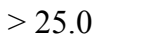 & $\square$ & $\square$ \\
\hline$\square \square \square \square$ & 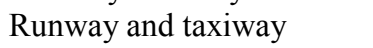 & पाणाम & $0.40<\mathrm{ETD} \leq 0.75 \square$ & $0.25 \leq \mathrm{ETD} \leq 0.40 \square$ & $\square$ & $\square$ & $\leq 0.25 \square$ \\
\hline $\begin{array}{l}\square \square \square \\
\square \square m \square\end{array}$ & 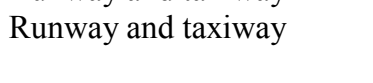 & $\begin{array}{l}\square \square \square \\
\square \square \square \square \square\end{array}$ & $\begin{array}{l}40<\mathrm{PCI} \leq 5 \overline{5} \\
\square \square\|\| \| \square \square\end{array}$ & $\begin{array}{l}25<\overline{\mathrm{PCI}} \leq 40 \\
\square \square\end{array}$ & 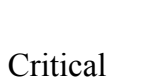 & $\square$ & $\leq 25 \square$ \\
\hline$\square \square \square \square \square$ & पामाणा & $\square$ & $\square$ & & & & \\
\hline
\end{tabular}

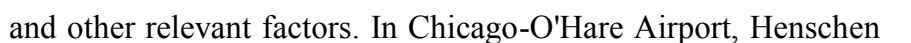

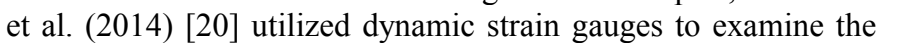

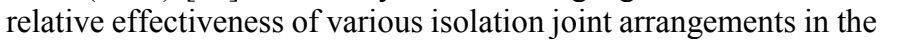

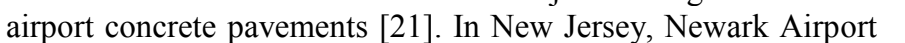

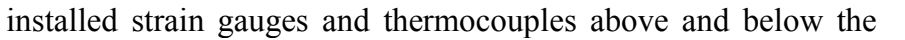

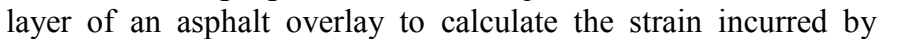

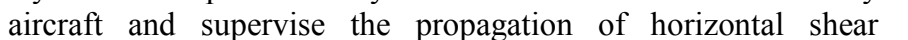

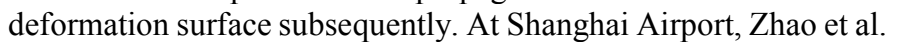

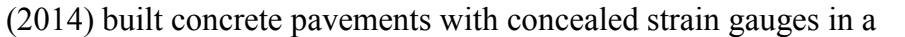

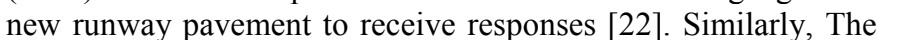

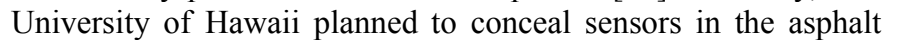

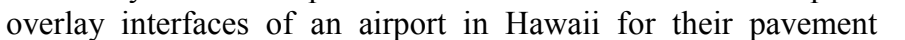

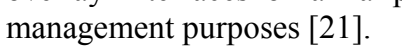

\section{GIS application}

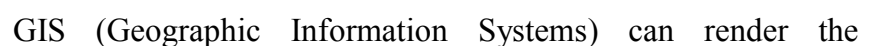

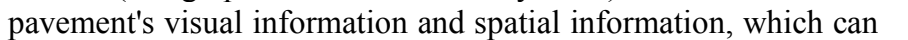

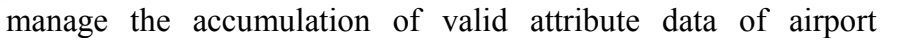

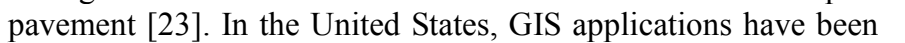

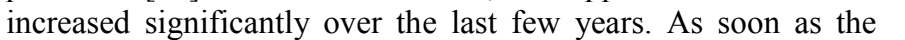

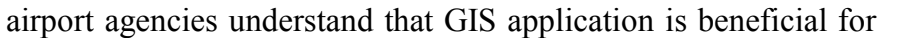

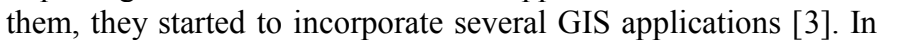

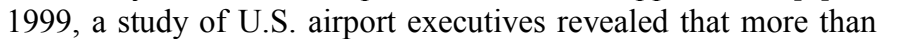

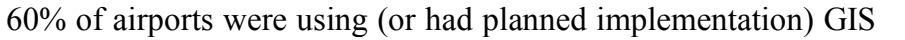

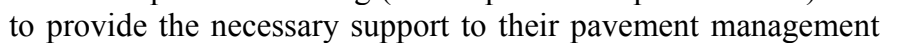

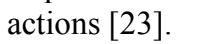

\subsubsection{Performance Models}

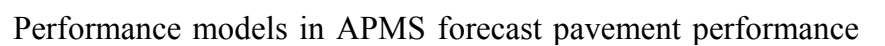

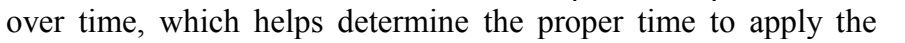

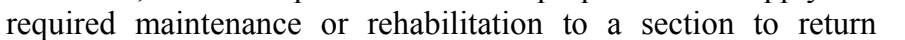

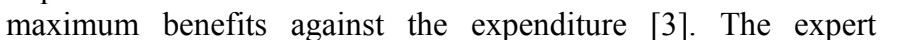

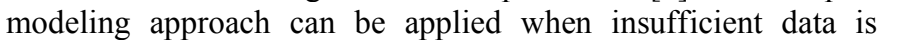

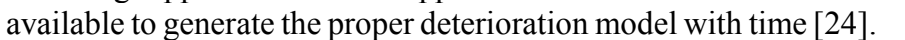

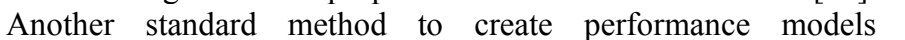

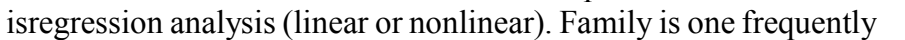

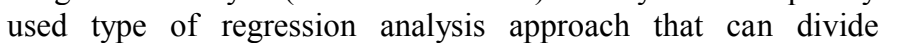

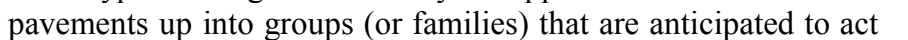

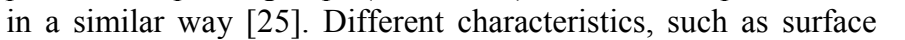

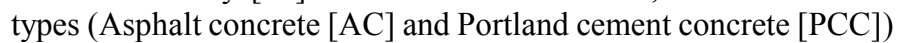

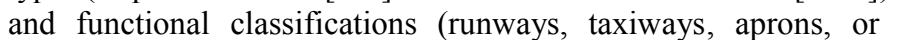

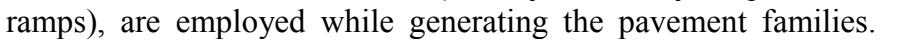

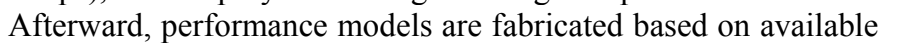

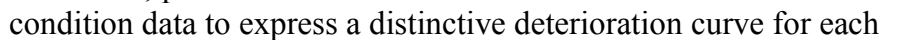
घण

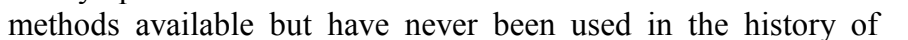

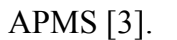

\subsection{Prioritization \& budgeting}

\subsubsection{Guidelines for prioritization}

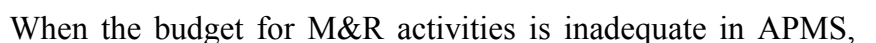

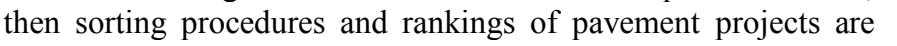

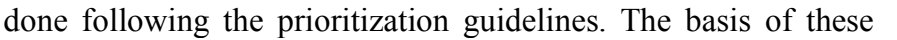

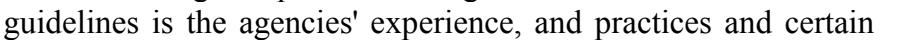

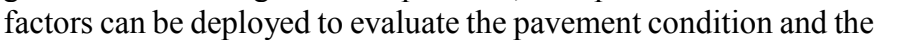

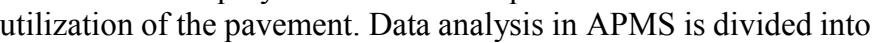

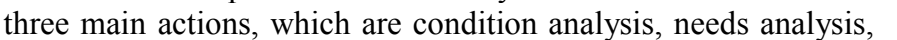

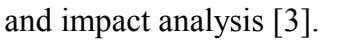

\section{Analyze the condition}

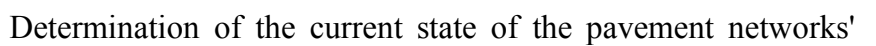

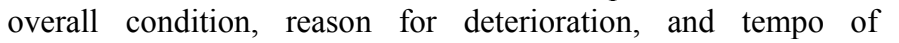

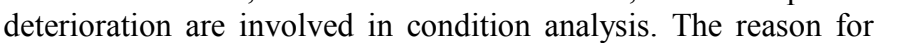

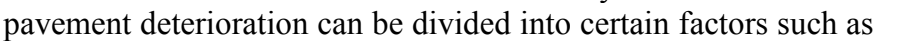

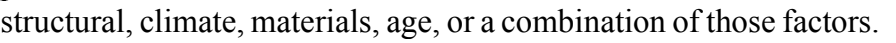

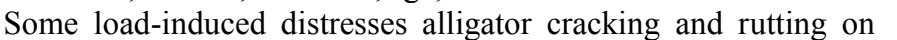

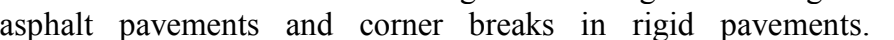

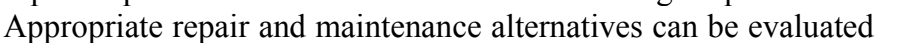

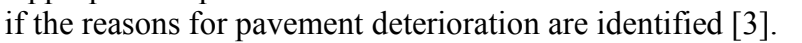

\section{Analysis of needs}

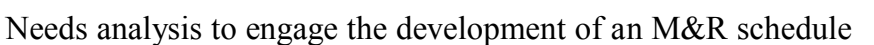

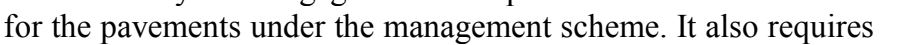

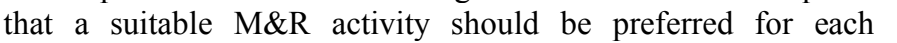

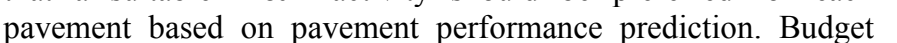

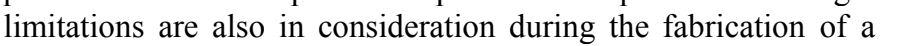
need's analysis; therefore, the M\&R actions must follow the

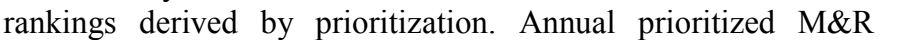

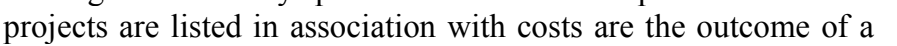
successful need's analysis [3].

\section{Impact analysis}

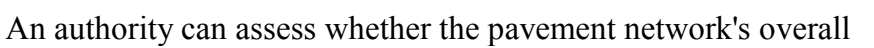

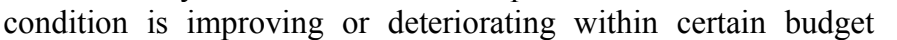

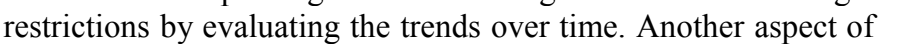

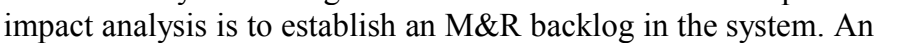

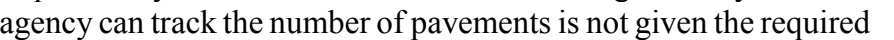

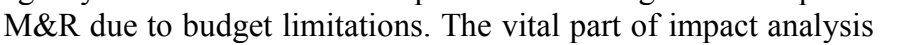

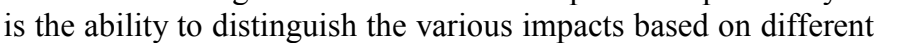

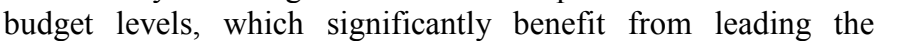

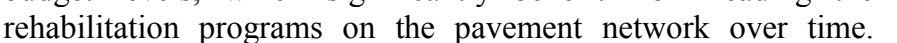

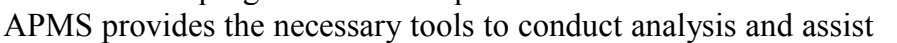

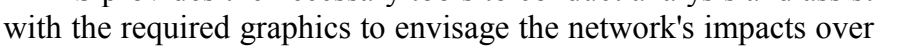

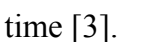




\subsubsection{Maintenance and rehabilitation policies in association with the costs}

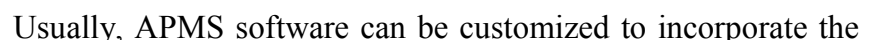

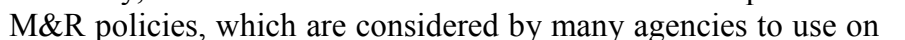
पा

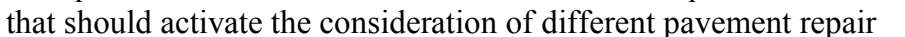

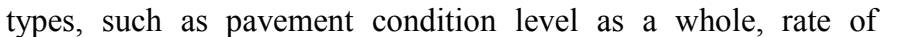

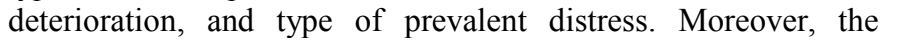

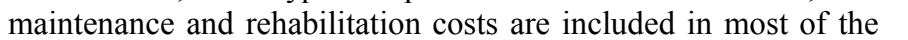

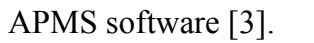

\subsubsection{Selection of alternative strategies}

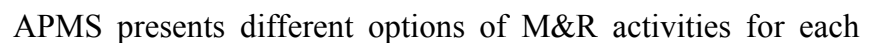

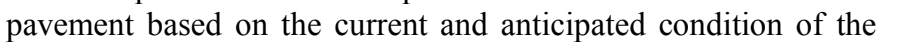

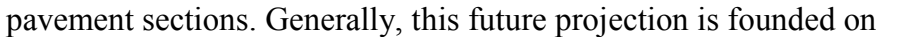

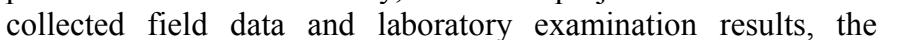

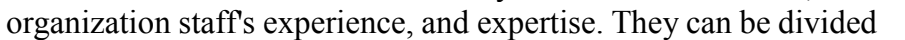
प

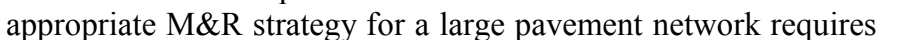

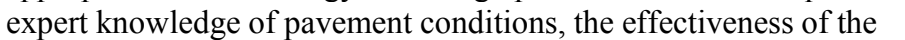

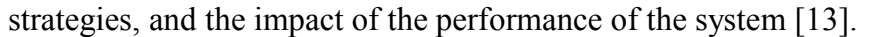

\section{Life cycle cost analysis (LCCA)}

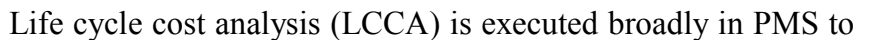

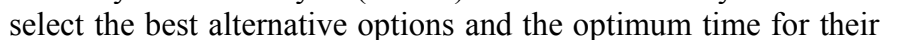

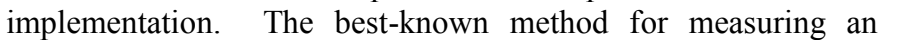

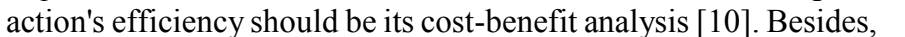

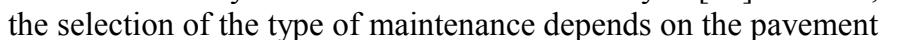

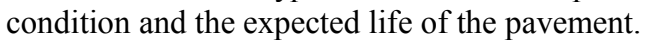

\subsection{APMS outputs}

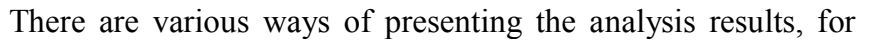

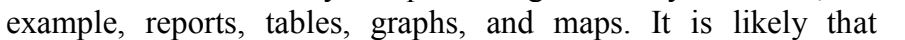

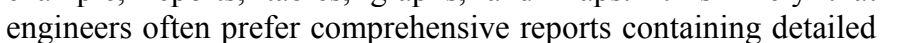

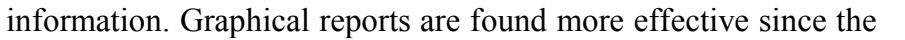

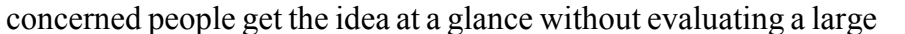

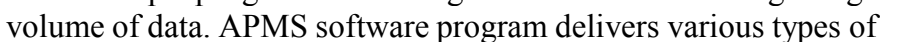
ए

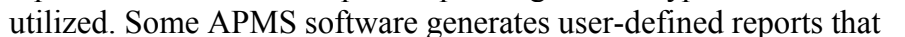

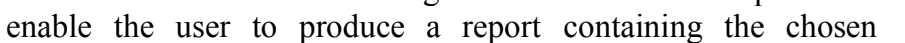
प

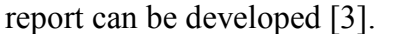

\subsection{Relevant issues in APMS implementations}

\subsubsection{Training process}

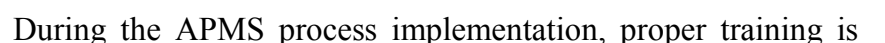

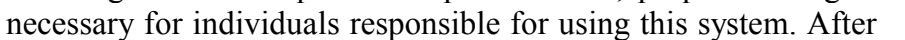

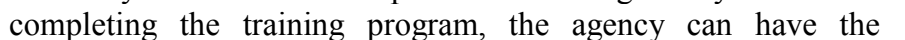

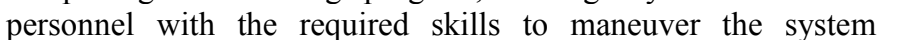

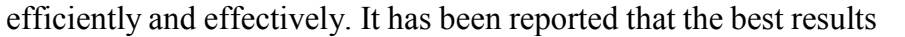

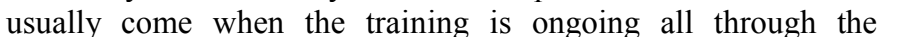

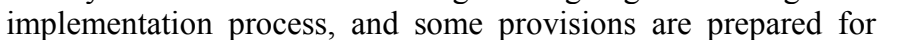

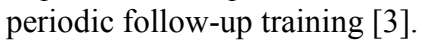

\subsubsection{Follow-up of APMS implementation process}

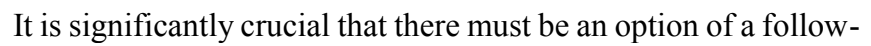

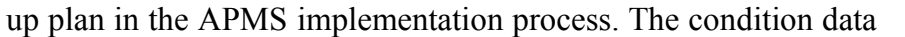

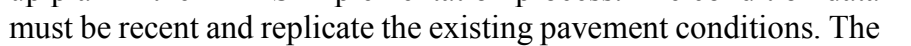

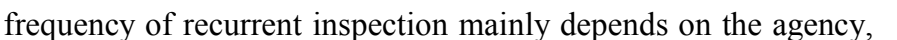

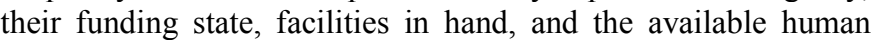

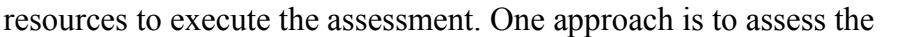

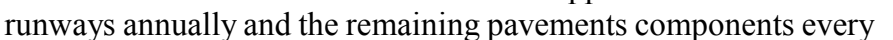

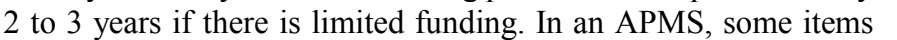

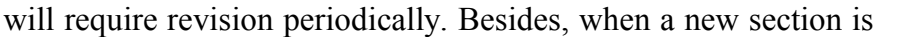

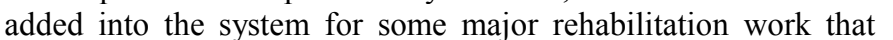

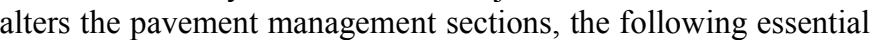

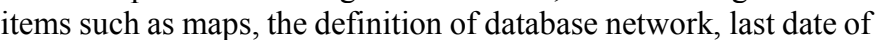

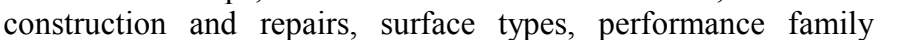

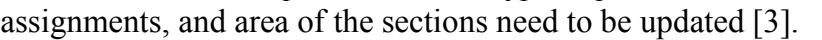

\subsubsection{Performance observation and prediction}

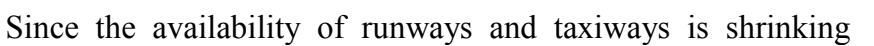

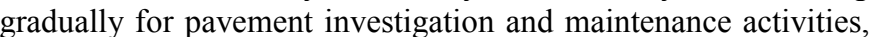

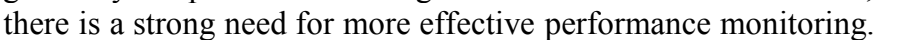

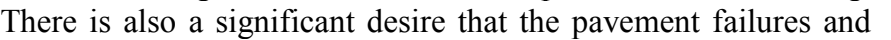

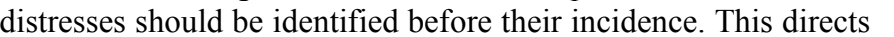

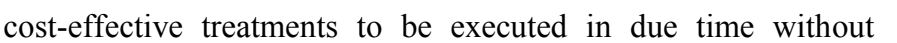

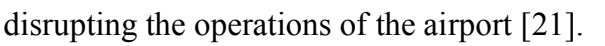

\section{APMS practice and issues if different airports}

\subsection{APMS in different airports in the USA}

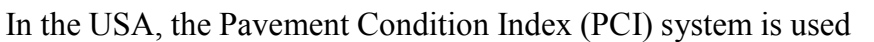

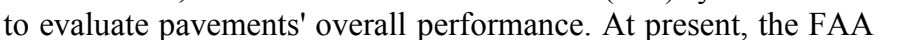

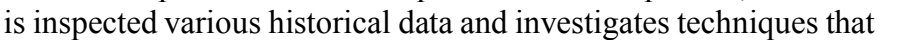

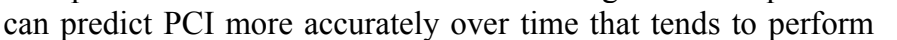

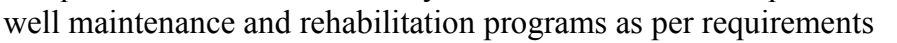
एणाणाए

प

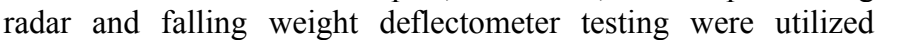

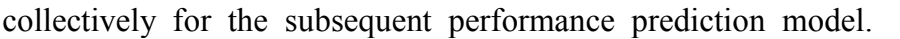

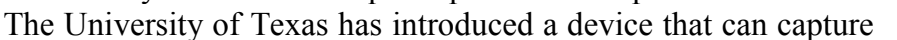

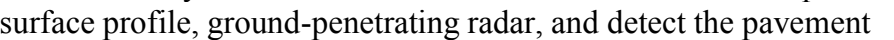

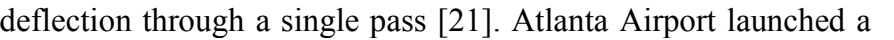

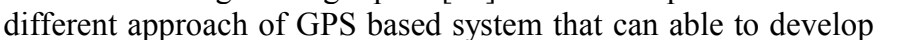

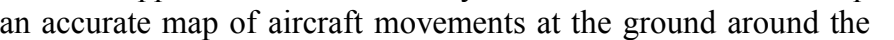

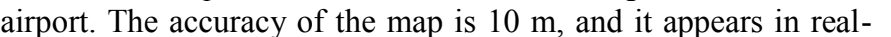

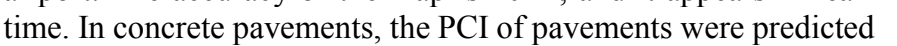

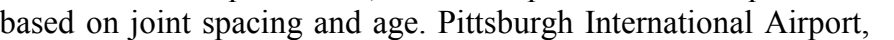

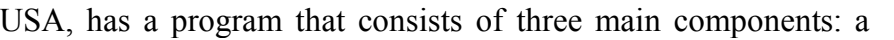

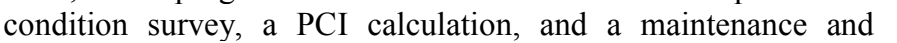

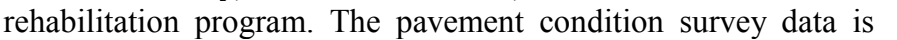

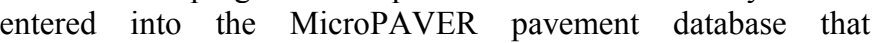

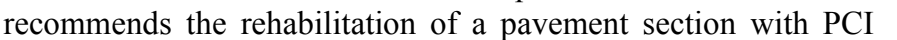

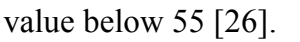

\subsection{APMS at Rome international airport}

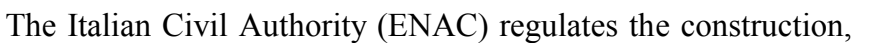

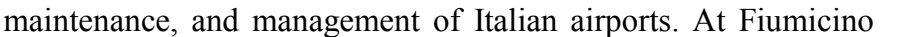

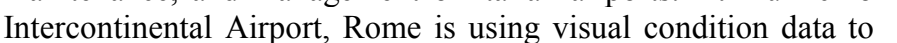

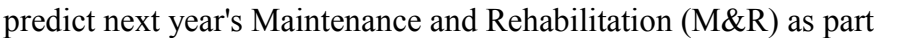

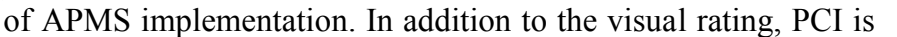

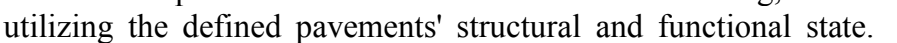

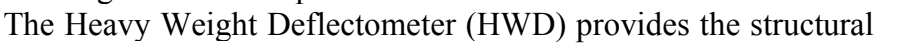

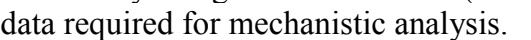

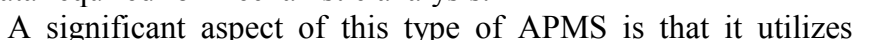

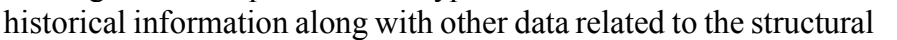

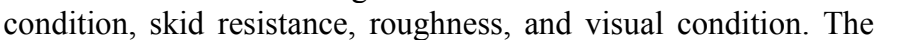




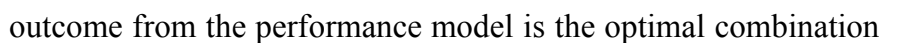

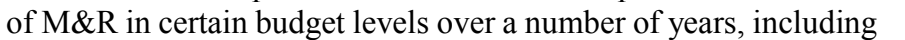
पा

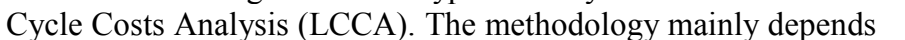

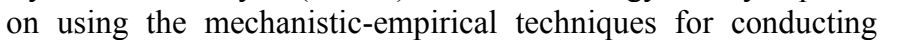

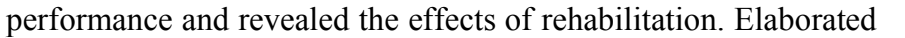

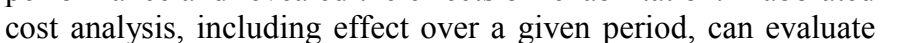

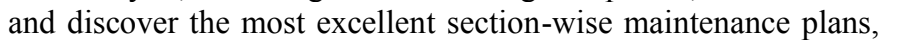

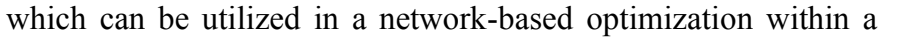

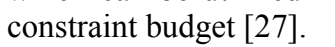

\subsection{APMS in Australia}

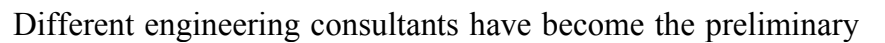

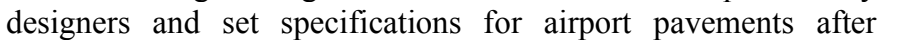

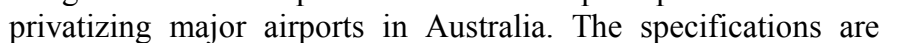

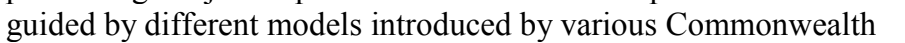

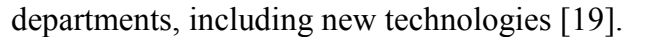

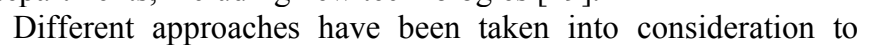

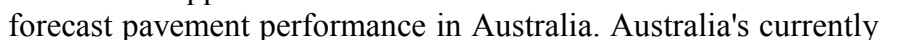

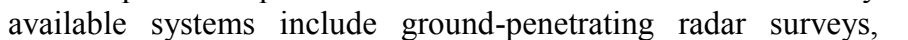

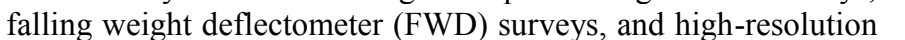

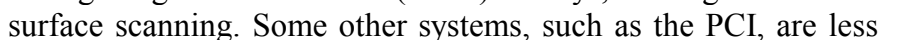

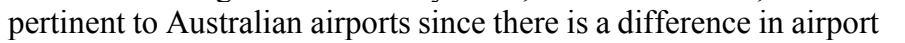

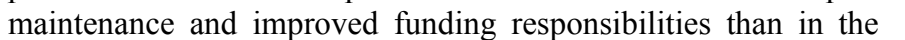

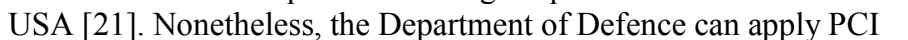

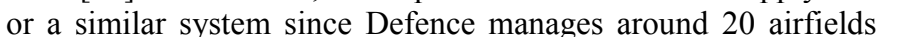

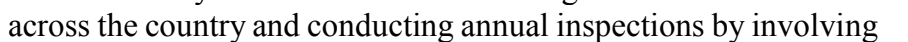

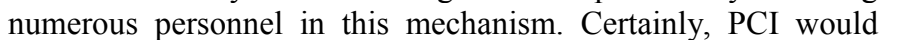

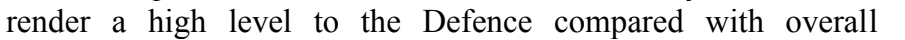

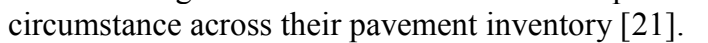

\subsection{GIS \& GPS application in Chinese Airport under SHAPMS}

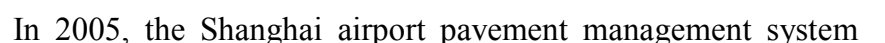

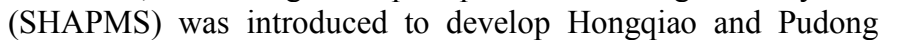

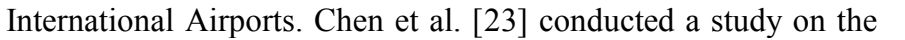

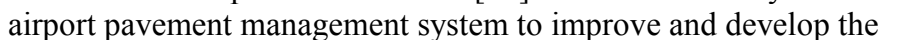

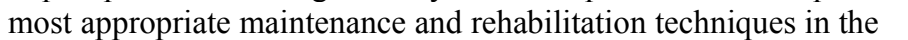

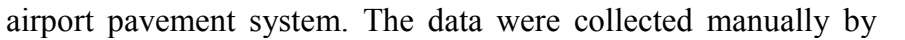

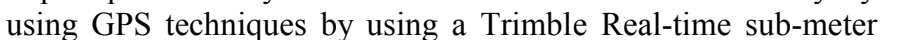

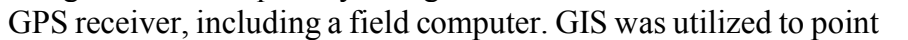

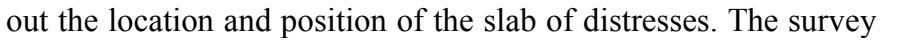

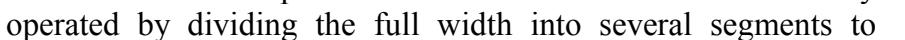

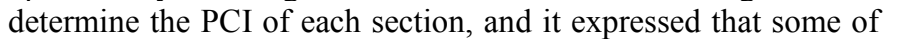

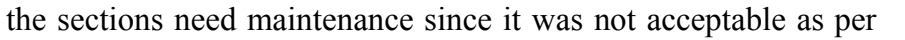

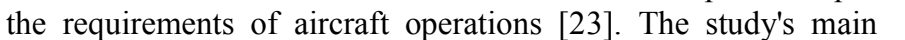

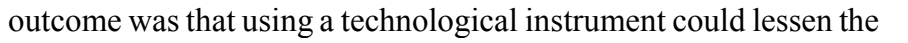

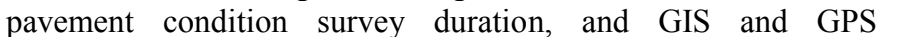

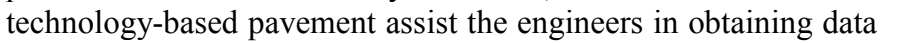

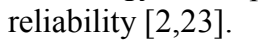

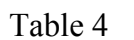

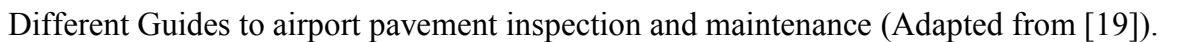

\subsection{Case studies on surface distress of airport pavements in Japan}

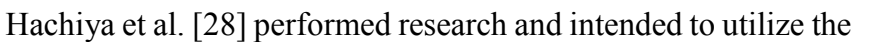

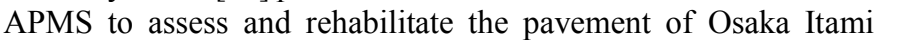

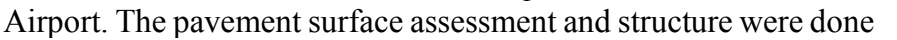

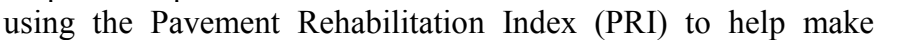

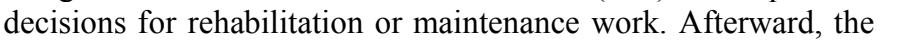

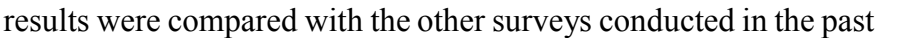

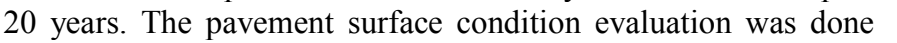

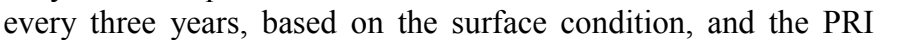

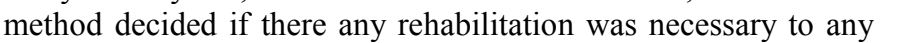

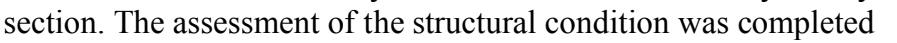
पाm

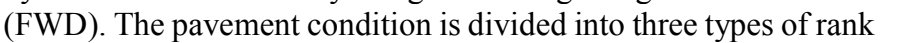

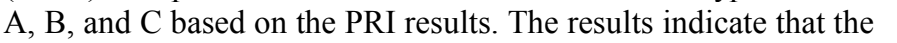

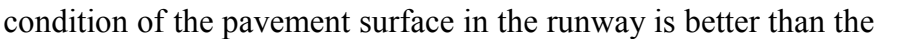

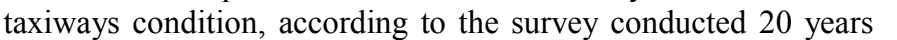

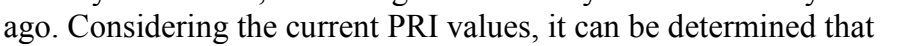

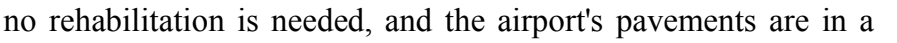

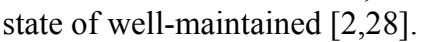

\section{Distresses}

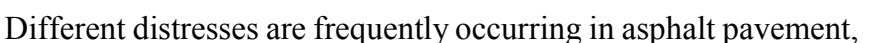

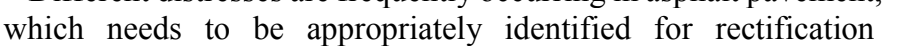

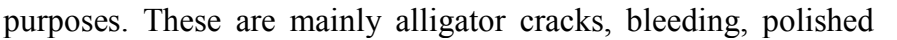

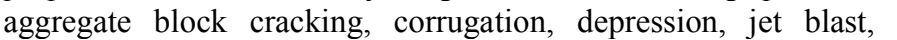

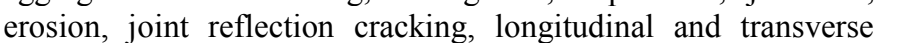

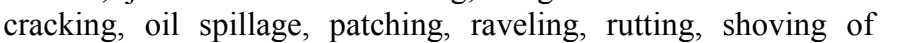

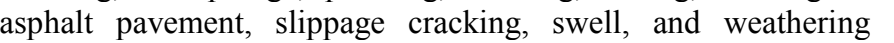

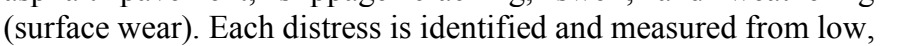

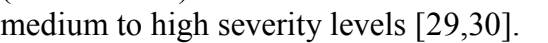

\section{Guidelines to distress and maintenance}

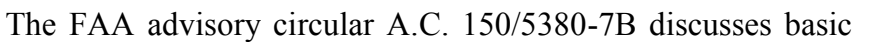

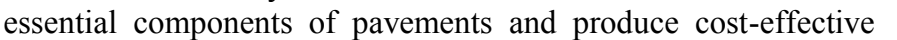

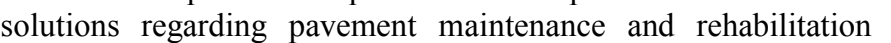

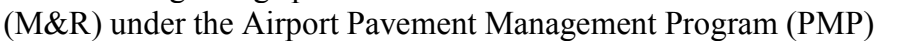

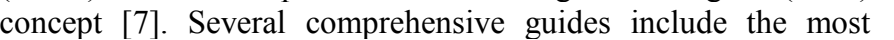

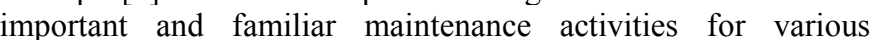

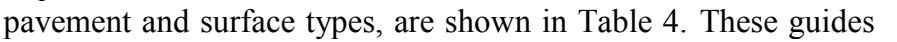

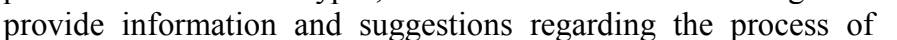

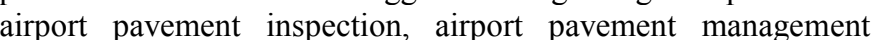

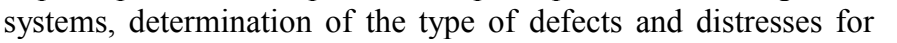

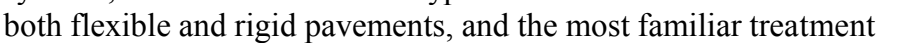

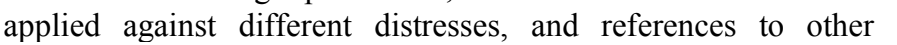

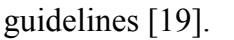

\begin{tabular}{|c|c|}
\hline 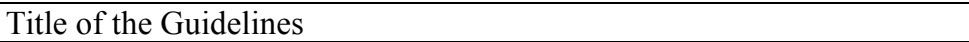 & 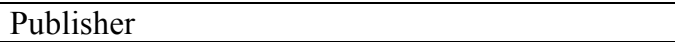 \\
\hline 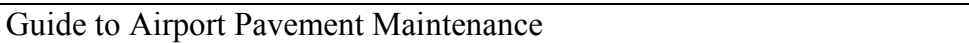 & 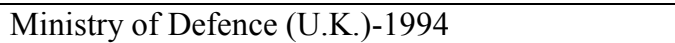 \\
\hline 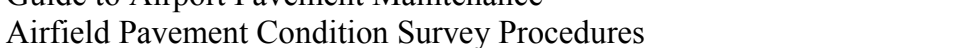 & 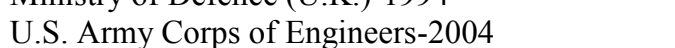 \\
\hline 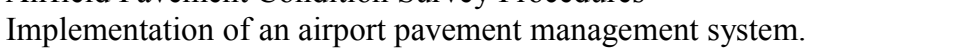 & 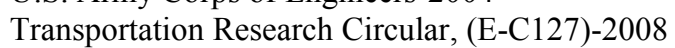 \\
\hline 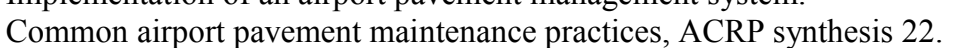 & 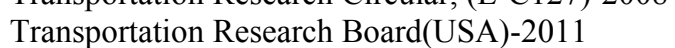 \\
\hline 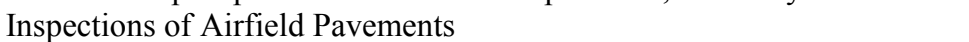 & 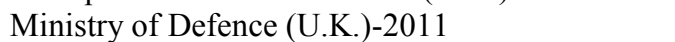 \\
\hline 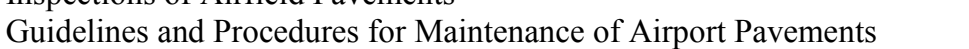 & 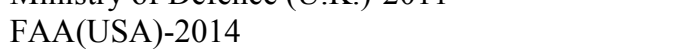 \\
\hline 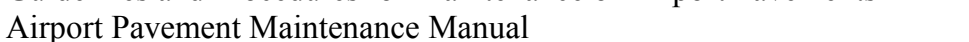 & 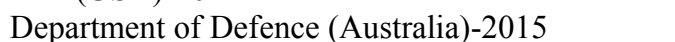 \\
\hline
\end{tabular}




\section{Expert system for APMS}

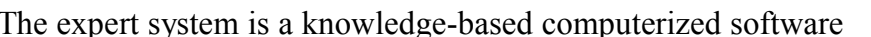

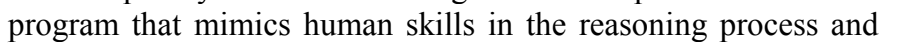

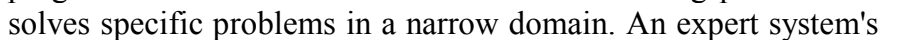

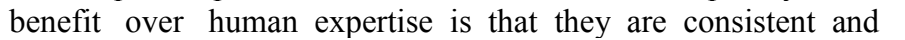

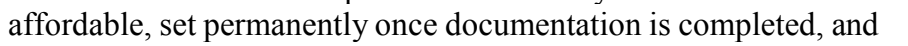

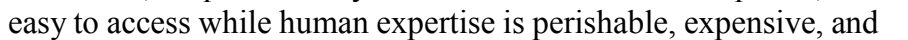

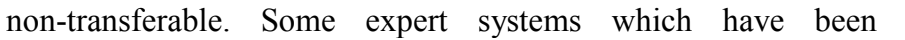

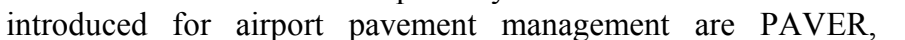
AIRPORTS, AIRPAVE, AIRPACS, AirScene ${ }^{\mathrm{TM}}$, and PAVEAIR.

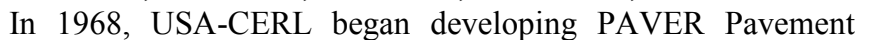

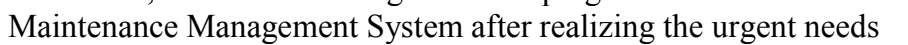

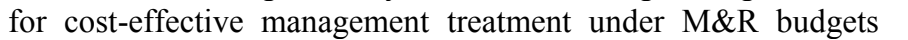

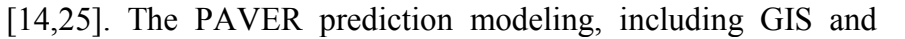

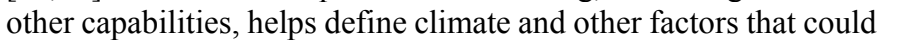

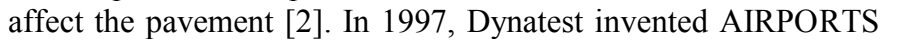

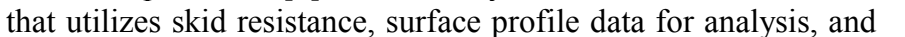

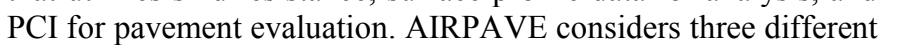

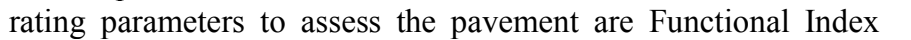
ए

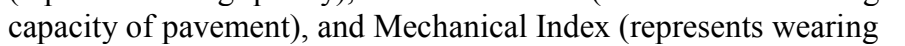

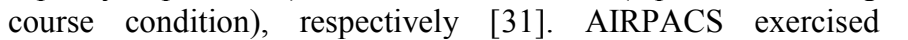

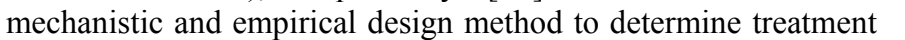

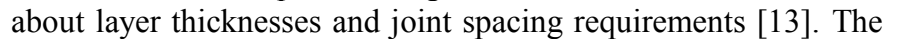
AirScene ${ }^{\mathrm{TM}}$ used to calculate the pavement damage generated by

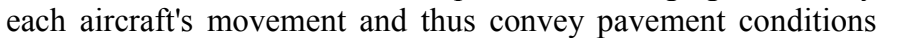

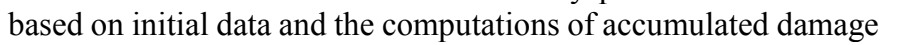

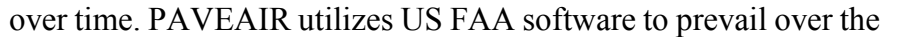

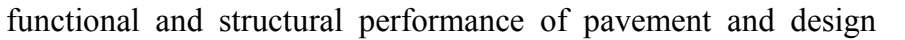

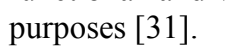

\section{Conclusion}

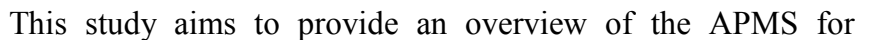

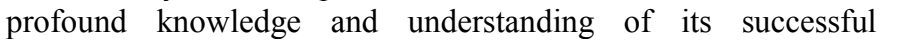

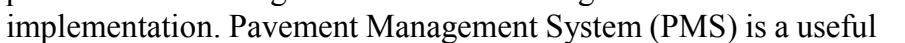

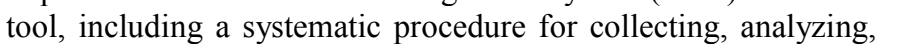

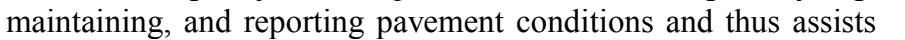

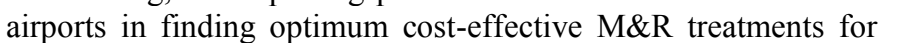

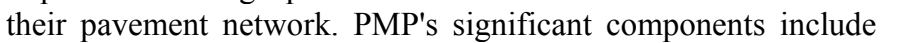

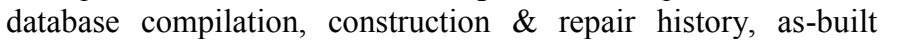

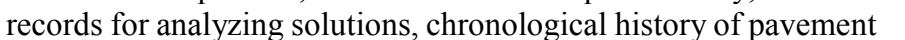

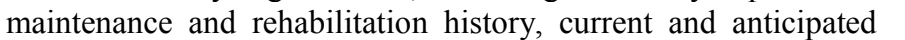
प

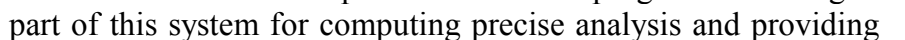

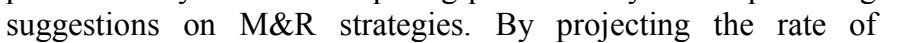

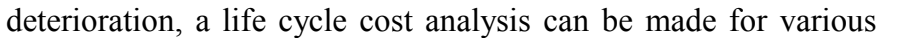

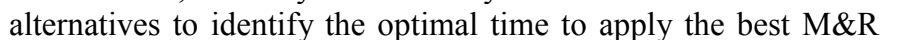

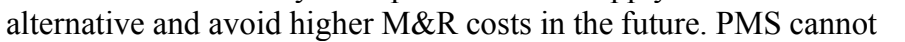

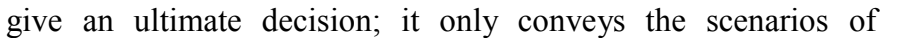

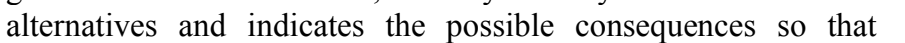

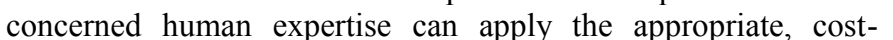

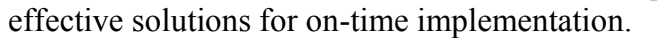

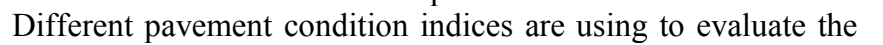

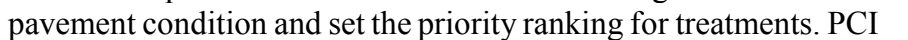

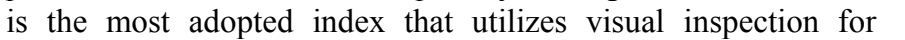

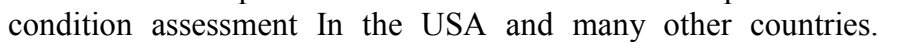

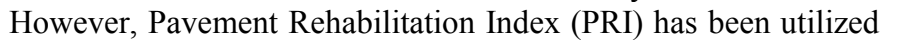

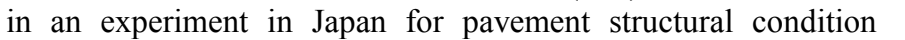

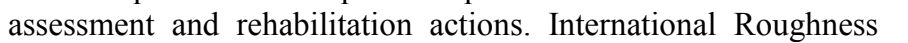

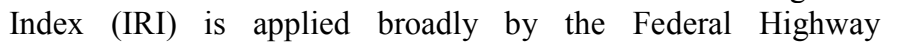

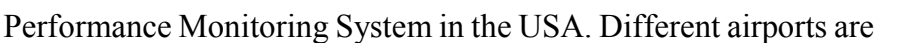

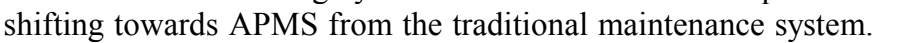

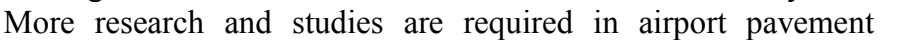

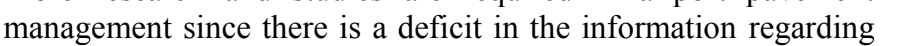

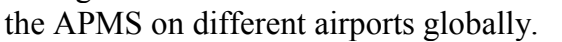

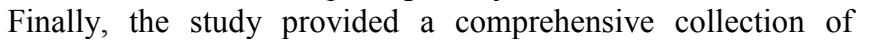

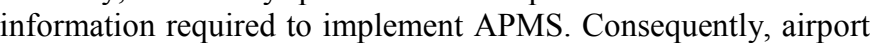

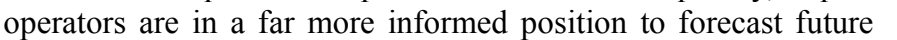

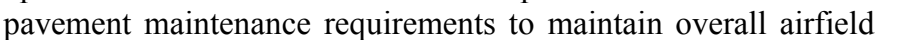

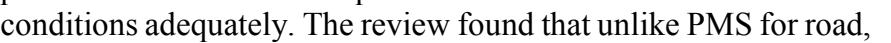

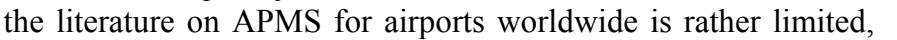

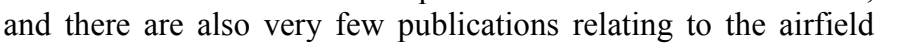

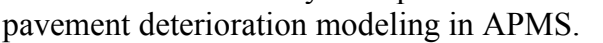

$\square$

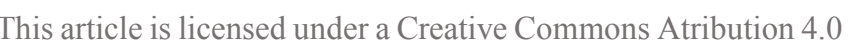

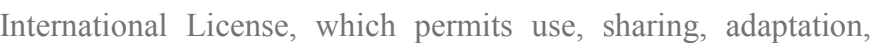

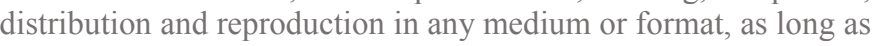

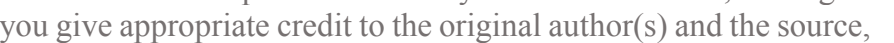

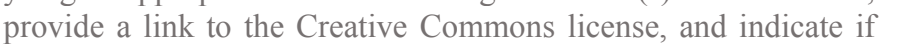

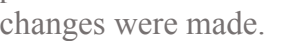

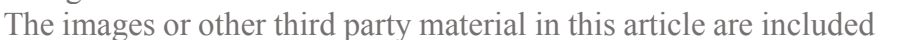

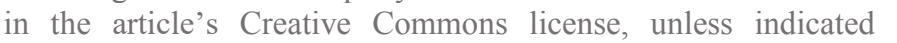

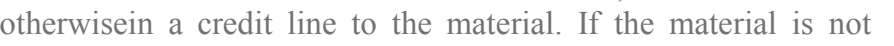

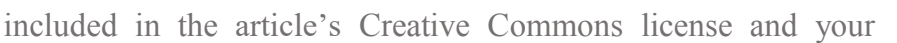

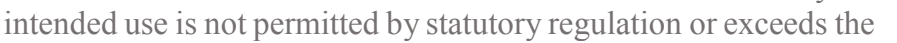

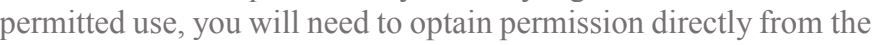

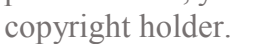

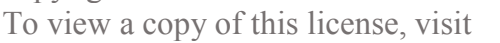

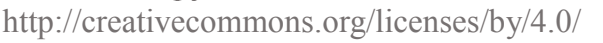

\section{Reference}

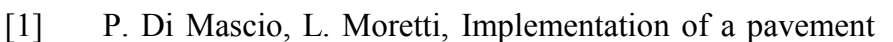

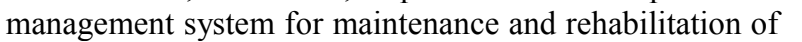

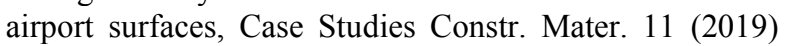
$\square 111 \mathrm{~m}$

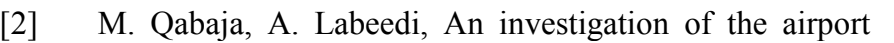

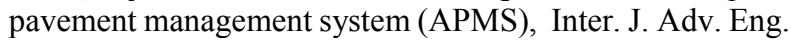

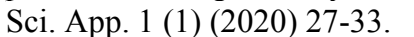

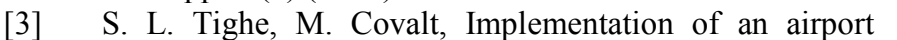

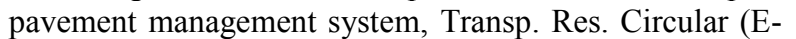

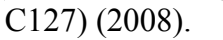

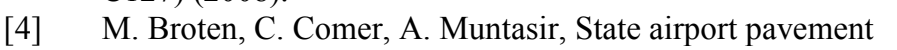

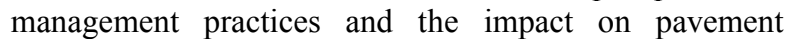

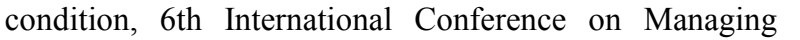

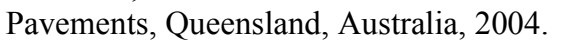

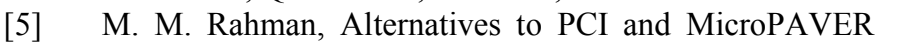

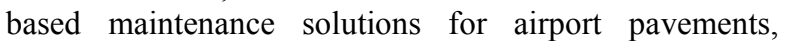

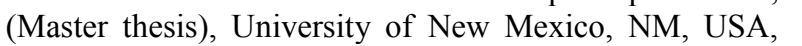

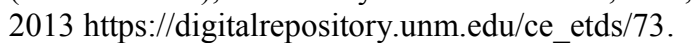

प1

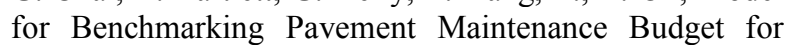

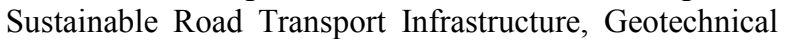

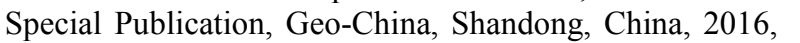
पाएापाणाण

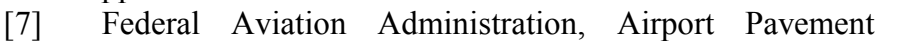

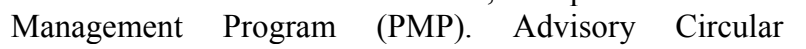

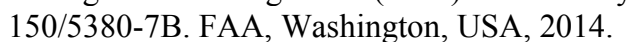

$\square \square$ S. G. de Oliveira, A. Tibaut, G. Dell'Acqua, Airport

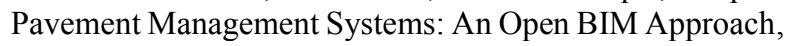

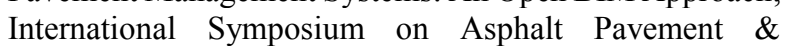

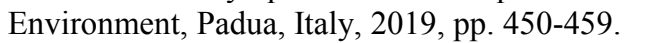




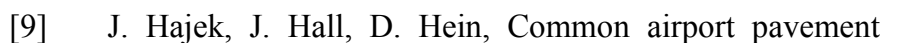

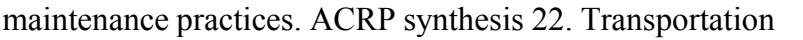

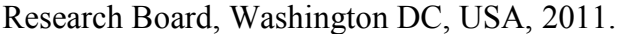

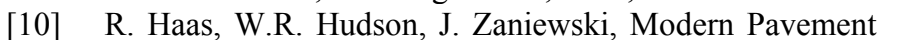

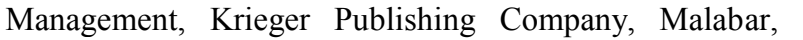

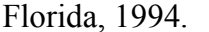

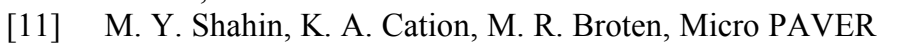

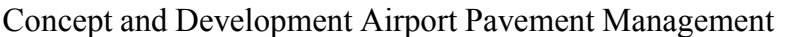

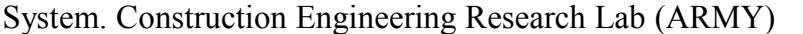

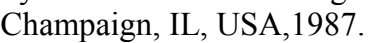

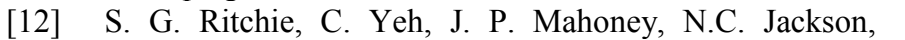

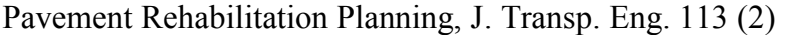

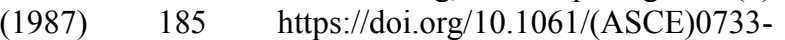

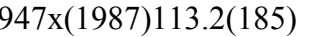

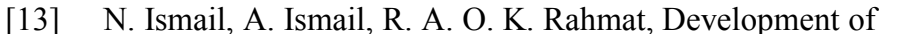

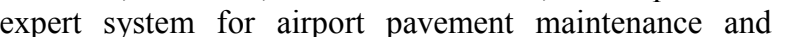

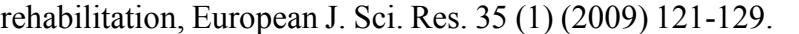

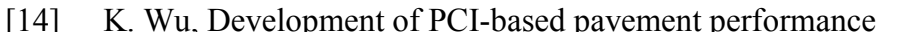

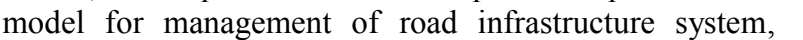

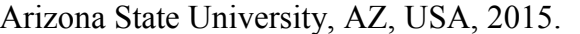

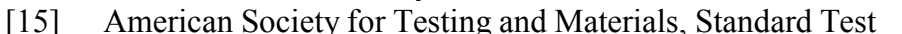

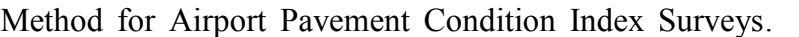

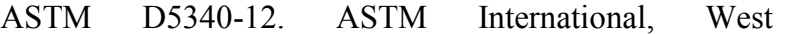

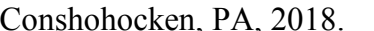

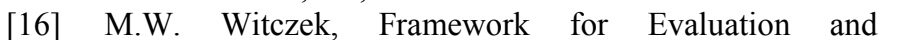

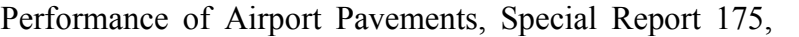

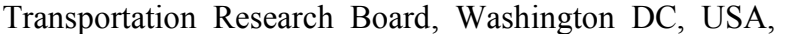
$\square|||||||||||| \mid \vec{~}$

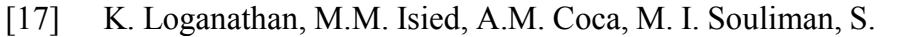

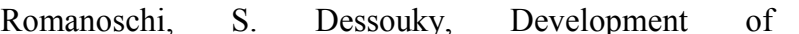

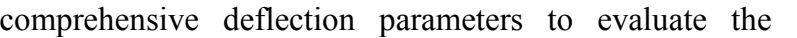

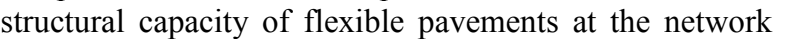

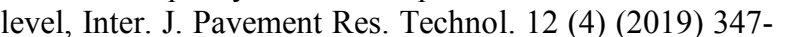

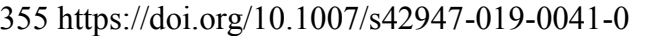

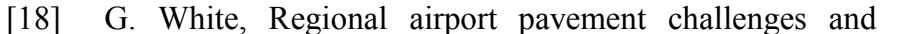

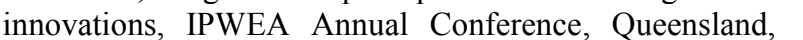

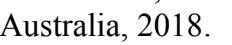

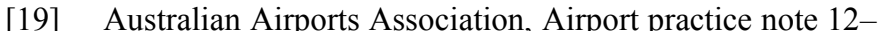

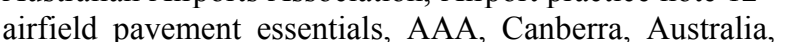
$\square 110$

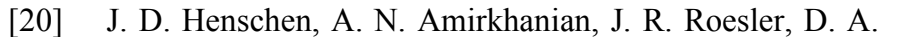

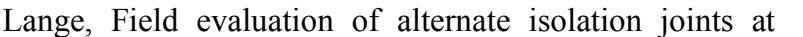
O'Hare international airport, Proceedings 2014 FAA

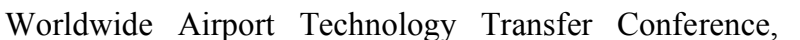

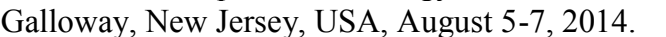

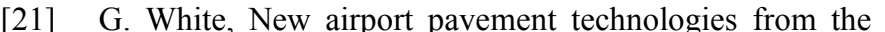

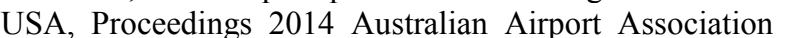

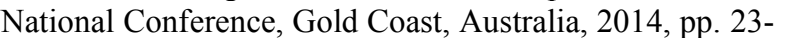
$\square \mathrm{IID}$

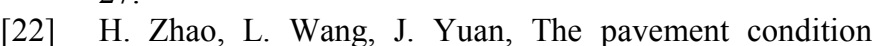

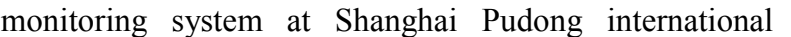

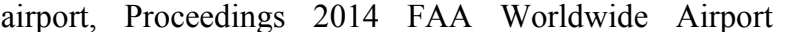

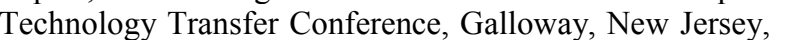

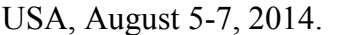

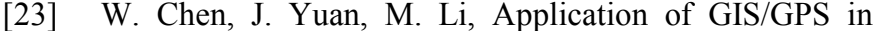

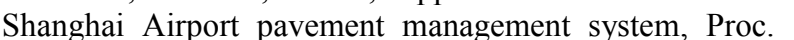

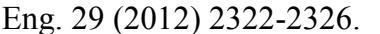

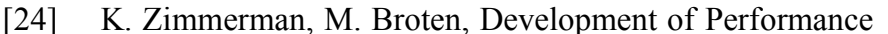

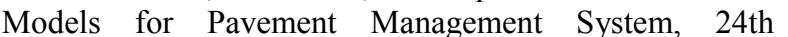

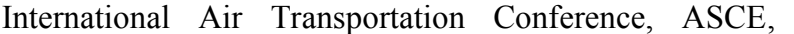

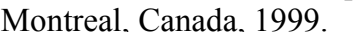

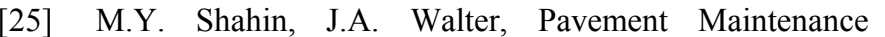

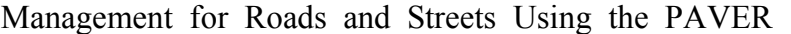

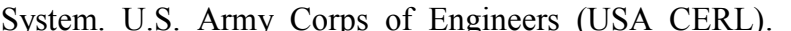

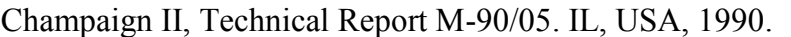

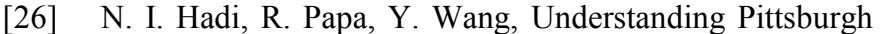

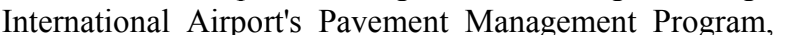

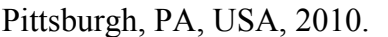

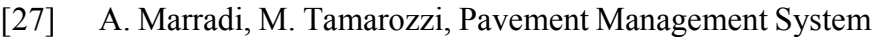

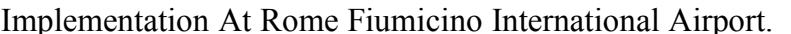

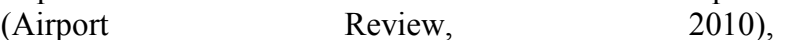

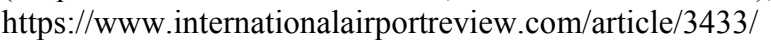

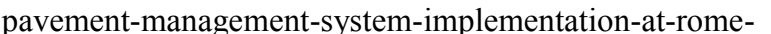

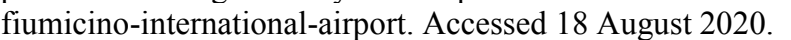

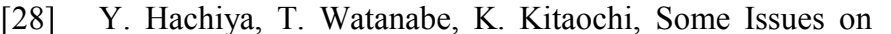

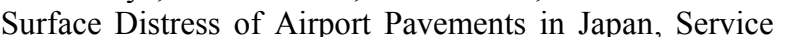

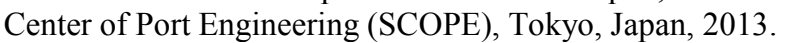

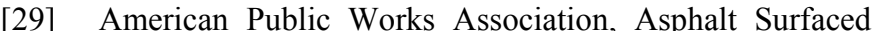

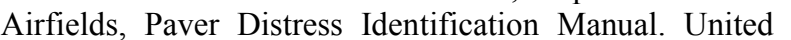

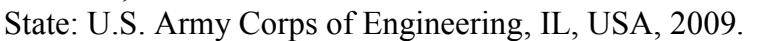

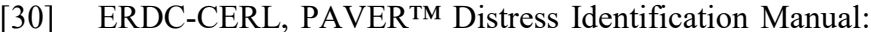

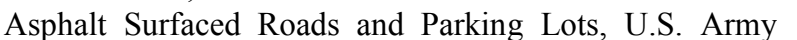

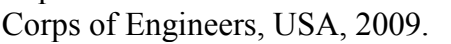

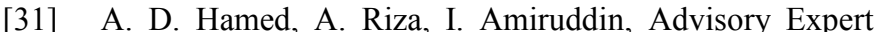

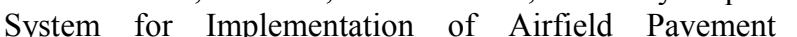

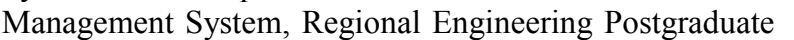

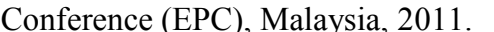

